\title{
A PENALTY METHOD FOR TOPOLOGY OPTIMIZATION SUBJECT TO A POINTWISE STATE CONSTRAINT
}

\author{
SAmuel Amstutz ${ }^{1}$
}

\begin{abstract}
This paper deals with topology optimization of domains subject to a pointwise constraint on the gradient of the state. To realize this constraint, a class of penalty functionals is introduced and the expression of the corresponding topological derivative is obtained for the Laplace equation in two space dimensions. An algorithm based on these concepts is proposed. It is illustrated by some numerical applications.
\end{abstract}

Mathematics Subject Classification. 49Q10, 49Q12, 49M30, 35J05.

Received January 8, 2008. Revised November 21, 2008.

Published online June 18, 2009.

\section{IntRoduction}

Mathematicians and engineers have at disposal various methods to address shape and topology optimization problems. They mainly rely on the following concepts: calculus of shape derivatives $[19,24,31,33]$, construction of relaxed formulations $[2,9,10]$, propagation of level sets $[4,7,35]$ and topological sensitivity analysis $[15,16,32]$. These methods have proven their efficiency to deal with academic or industrial problems in many fields of applications, such as structural optimization, design of electromagnetic components, shape control of fluids, and shape reconstruction from measurements. The treated problems are generally unconstrained or subject to a small number of constraints, like a volume constraint. Yet, in structural optimization for instance, the failure criteria generally involve spatial functions whose value at every point of the structure has to fulfill given requirements. This gives rise to shape and topology optimization problems subject to infinitely many inequality constraints. These problems, which are investigated in the present paper, concentrate several difficulties. Firstly, pointwise state constraints are known to be delicate to handle, even in the classical framework of the control by a function. One has to face a low regularity of the Lagrange multiplier, which is usually only a Borel measure, making multiplier based algorithms hardly directly applicable. Possible cures have been devised only recently $[20,22,23]$. Secondly, relaxing such problems seems to have no simple solution due to the local nature of the constraint. Unless this latter is weakened like in [3], the proposed strategies lead to tremendous computational efforts $[12,14]$. Finally, the set of definition of the constraint functional is deformed with the design domain. In the related context of generalized semi-infinite programming, solution methods have been developed only when the number of decision variables is finite and the problem has a special structure $[28,29,34]$.

\footnotetext{
Keywords and phrases. Topology optimization, topological derivative, penalty methods, pointwise state constraints.

${ }^{1}$ Laboratoire d'Analyse Non Linéaire et Géométrie, Faculté des Sciences, 33 rue Louis Pasteur, 84000 Avignon, France.

samuel.amstutz@univ-avignon.fr
} 
For all these reasons, we opt for the use of a penalty method associated with a topological derivative based algorithm. The advantage of the topological derivative is to allow topology variations without relaxation. We recall that the principle consists in analyzing the behavior of the objective functional with respect to topology perturbations, typically the nucleation of small holes in the domain. Penalty methods do not involve any Lagrange multiplier, and penalty functionals defined in the variable domain can be constructed in a natural way. Our framework is that of second order elliptic state equations. Among them, the linear elasticity equations with a constraint on the principal stresses, like the Von Mises stress, are a target of major interest for the applications. However, the multidimensionality of this system raises technical difficulties which we want to avoid in a first step. Therefore, we focus in this paper on the scalar Laplace equation, whose solutions exhibit a similar behavior near geometric singularities. By analogy with the principal stresses, we choose a constraint acting on the gradient of the state. Using an adjoint method, we carry out the topological sensitivity analysis of a class of smooth penalty functionals. Because such functionals do not allow for an exact penalization, we construct a sequence of functionals from this class and use them within an iterative algorithm. Convergence properties, which are always very hard to study whenever shapes are involved, are not investigated at the theoretical level, but they are illustrated by some numerical experiments.

The paper is organized as follows. The model problem and the class of penalty functionals under consideration are presented in Section 2. The topological sensitivity analysis of these functionals is carried out in Sections 3 and 4. The main result is stated in the end of the latter. A part of the proofs is gathered in Section 5 for readability. The penalty functionals used in the computations are exhibited in Section 6, and the algorithm is described in Section 7. Section 8 is dedicated to the numerical examples and concludes as to the efficiency of the method.

\section{Problem statement}

\subsection{The constrained problem}

Let $D$ be a bounded domain (connected open subset) of $\mathbb{R}^{2}$ with a Lipschitz boundary $\Gamma$ made of two disjoint parts $\Gamma_{D}$ and $\Gamma_{N}, \Gamma_{D}$ being of nonzero measure and $\Gamma_{N}$ being of class $\mathcal{C}^{1}$. Let $\mathcal{E}$ be a set of subdomains of $D$. For all $\Omega \in \mathcal{E}$ we define the piecewise constant function

$$
\alpha_{\Omega}=\left\{\begin{array}{rll}
\alpha_{\text {in }} & \text { in } & \Omega \\
\alpha_{\text {out }} & \text { in } & D \backslash \bar{\Omega}
\end{array}\right.
$$

where $\alpha_{\text {in }}$ and $\alpha_{\text {out }}$ are different positive numbers. In the applications, $D \backslash \bar{\Omega}$ will be occupied by a phase with very low conductivity meant to approximate an empty region. Hence there will hold

$$
\frac{\alpha_{\text {out }}}{\alpha_{\text {in }}} \ll 1
$$

Given a distribution $g \in H_{00}^{1 / 2}\left(\Gamma_{N}\right)^{\prime}$, we denote by $u_{\Omega} \in H^{1}(D)$ the solution of the boundary value problem:

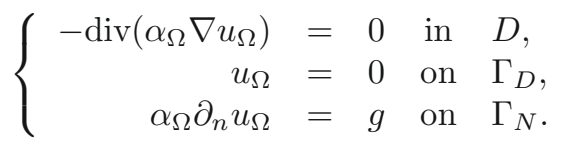

We want to minimize over $\mathcal{E}$ an objective functional of the form $\Omega \mapsto I_{\Omega}\left(u_{\Omega}\right)$. In addition, given a 2 by 2 symmetric positive semidefinite matrix $B$, an open set $\tilde{D} \subset D$ and a positive constant $M$, we want to realize the constraint

$$
\frac{1}{2} B \nabla u_{\Omega} \cdot \nabla u_{\Omega} \leq M \quad \text { a.e. in } \Omega \cap \tilde{D} .
$$


In summary, the problem under investigation reads:

$$
\min _{\Omega \in \mathcal{E}} I_{\Omega}\left(u_{\Omega}\right) \quad \text { subject to }(2.2) \text { and }(2.3) \text {. }
$$

Due to the lack of convexity property, we will content ourselves with local minimizers. The class of perturbations used to characterize these domains will be specified in Section 3.1.

\subsection{Penalized formulation}

Given a positive parameter $\gamma$, we consider the penalized objective functional

$$
I_{\Omega}^{\gamma}\left(u_{\Omega}\right)=I_{\Omega}\left(u_{\Omega}\right)+\gamma J_{\Omega}\left(u_{\Omega}\right)
$$

where the penalty functional $J_{\Omega}$ is of the form

$$
\begin{gathered}
J_{\Omega}(u)=\int_{\tilde{D}} \beta_{\Omega} \Phi\left(\frac{1}{2} B \nabla u \cdot \nabla u\right) \mathrm{d} x, \\
\beta_{\Omega}=\left\{\begin{array}{lll}
\beta_{\text {in }} & \text { in } & \Omega, \\
\beta_{\text {out }} & \text { in } & D \backslash \bar{\Omega} .
\end{array}\right.
\end{gathered}
$$

The above coefficients $\beta_{\text {in }}$ and $\beta_{\text {out }}$ are constants, ideally $\beta_{\text {out }}=0$ (however there will be a little restriction, see Rem. 2.1). The function $\Phi: \mathbb{R}_{+} \rightarrow \mathbb{R}_{+}$is assumed to be nondecreasing with at most linear growth at infinity, which allows to define $J_{\Omega}(u)$ for all $u \in H^{1}(D)$. Although $u_{\Omega}$ generally enjoys higher regularity, but not much higher if the domain $D$ has corners, this growth condition will be nevertheless useful for our analysis. For the sake of readability, we denote $\Phi\left(\frac{1}{2} B \nabla u \cdot \nabla u\right)$ instead of $\Phi \circ\left(\frac{1}{2} B \nabla u \cdot \nabla u\right)$. The penalized problem reads:

$$
\min _{\Omega \in \mathcal{E}} I_{\Omega}^{\gamma}\left(u_{\Omega}\right) \quad \text { subject to }(2.2) .
$$

In general, problems (2.4) and (2.7) are not equivalent. More precisely, the penalization is said to be exact at a local minimizer $\Omega^{\sharp}$ of (2.4) if $\Omega^{\sharp}$ is a local minimizer of (2.7). We expect that, like in nonlinear programming, exactness can be generically achieved only if the penalty functional is non-differentiable (see e.g. [11]). However, to enable the resolution of (2.7) by using the topological derivative, the function $\Phi$ has to be smooth (at least $\left.\mathcal{C}^{1}\right)$. This difficulty can be overcome by two ways:

(1) Construct a sequence $\left(\gamma_{n}\right)_{n \in \mathbb{N}}$ with $\lim _{n \rightarrow+\infty} \gamma_{n}=+\infty$, then solve (2.7) with $\gamma_{n}$ in place of $\gamma$ for increasing values of $n$.

(2) Construct a sequence of smooth penalty functions $\left(\Phi_{n}\right)_{n \in \mathbb{N}}$ tending to a presumed exact (non-differentiable) penalty function $\Phi_{\infty}$, then solve (2.7) with $\gamma$ fixed in advance and $\Phi_{n}$ in place of $\Phi$ for increasing values of $n$.

We choose the second strategy, although the first one is far more common. This choice is justified by the fact that the growth condition imposed on $\Phi$ prohibits the use of the classical quadratic penalty function $t \mapsto \max (t-M, 0)^{2}$. We point out that this phenomenon is peculiar to nonsmooth domains, which we want to be able to address (see Rem. 4.4). In the shape sensitivity framework with smooth boundaries, the quadratic penalty function is used e.g. in [21].

An example of suitable sequence of penalty functions is given in Section 6. For the moment, we assume that $\Phi$ and $\gamma$ are given.

Remark 2.1. We will see that the subsequent analysis requires the condition

$$
\frac{\alpha_{\text {out }}}{\alpha_{\text {in }}}=\frac{\beta_{\text {out }}}{\beta_{\text {in }}} .
$$


Hence $\beta_{\text {out }}$ cannot be equal to zero. Nevertheless, in view of (2.1), this limitation results only in a very minor alteration of the initial problem.

\section{FRAMEWORK FOR THE TOPOLOGICAL SENSITIVITY ANALYSIS}

\subsection{Topology perturbations}

In order to apply a topology optimization algorithm to problem (2.7) such as those proposed in [5,7,13,16,26], we need to know the expression of the topological derivative of the functional $I_{\Omega}^{\gamma}\left(u_{\Omega}\right)$. We assume that the topological derivative of the objective functional $I_{\Omega}\left(u_{\Omega}\right)$ is known. Therefore the theoretical part of this paper is focused on the topological sensitivity analysis of the penalty functional $J_{\Omega}\left(u_{\Omega}\right)$.

Starting from a current domain $\Omega \subset D$, we consider two kinds of perturbation: the creation of a hole inside $\Omega$ and the nucleation of a new connected component to $\Omega$. Given a smooth bounded domain $\omega \subset \mathbb{R}^{2}$, a point $x_{0} \in D \backslash \partial \Omega$ and a parameter $\varepsilon>0$, we denote by $\omega_{\varepsilon}=x_{0}+\varepsilon \omega$ the shifted and rescaled image of $\omega$. In this paper, we only consider circular perturbations, i.e. $\omega=B(0,1), \omega_{\varepsilon}=B\left(x_{0}, \varepsilon\right)$. The new design domain $\Omega_{\varepsilon}$ is defined by

$$
\Omega_{\varepsilon}=\left\{\begin{array}{lll}
\Omega \backslash \overline{\omega_{\varepsilon}} & \text { if } & x_{0} \in \Omega, \\
\Omega \cup \omega_{\varepsilon} & \text { if } & x_{0} \in D \backslash \bar{\Omega} .
\end{array}\right.
$$

It is clear that $\omega_{\varepsilon}$ is contained either in $\Omega$ or in $D \backslash \bar{\Omega}$ provided that $\varepsilon$ is small enough.

We assume from now on that $x_{0}$ is fixed and, for notational simplicity, we denote $\left(\alpha_{\varepsilon}, \beta_{\varepsilon}, u_{\varepsilon}, J_{\varepsilon}\right)$ instead of $\left(\alpha_{\Omega_{\varepsilon}}, \beta_{\Omega_{\varepsilon}}, u_{\Omega_{\varepsilon}}, J_{\Omega_{\varepsilon}}\right)$ and $\left(\alpha_{0}, \beta_{0}, u_{0}, J_{0}\right)$ instead of $\left(\alpha_{\Omega}, \beta_{\Omega}, u_{\Omega}, J_{\Omega}\right)$. The state equations and the penalty functional can be rewritten as:

$$
\begin{aligned}
& \left\{\begin{array}{rllll}
-\operatorname{div}\left(\alpha_{\varepsilon} \nabla u_{\varepsilon}\right) & = & 0 & \text { in } & D, \\
u_{\varepsilon} & = & 0 & \text { on } & \Gamma, \\
\alpha_{\varepsilon} \partial_{n} u_{\varepsilon} & = & g & \text { on } & \Gamma_{N},
\end{array}\right. \\
& J_{\varepsilon}(u)=\int_{\tilde{D}} \beta_{\varepsilon} \Phi\left(\frac{1}{2} B \nabla u \cdot \nabla u\right) \mathrm{d} x .
\end{aligned}
$$

In all the sequel, we suppose that $\varepsilon$ does not exceed 1 . Then, for both types of perturbations, $\alpha_{\varepsilon}$ and $\beta_{\varepsilon}$ can be expressed as:

$$
\alpha_{\varepsilon}(x)=\left\{\begin{array}{ll}
\alpha_{0}(x) & \text { if } x \in D \backslash \overline{\omega_{\varepsilon}}, \\
\alpha_{1}(x) & \text { if } x \in \omega_{\varepsilon},
\end{array} \quad \beta_{\varepsilon}(x)= \begin{cases}\beta_{0}(x) & \text { if } x \in D \backslash \overline{\omega_{\varepsilon}} \\
\beta_{1}(x) & \text { if } x \in \omega_{\varepsilon}\end{cases}\right.
$$

It stems from the previous assumptions that $\alpha_{0}, \alpha_{1}, \beta_{0}$ and $\beta_{1}$ are constant in a neighborhood of $x_{0}$. We denote by $\alpha_{0}^{*}, \alpha_{1}^{*}, \beta_{0}^{*}$ and $\beta_{1}^{*}$ the corresponding values, namely $\left(\alpha_{0}^{*}, \beta_{0}^{*}, \alpha_{1}^{*}, \beta_{1}^{*}\right)=\left(\alpha_{\text {in }}, \beta_{\text {in }}, \alpha_{\text {out }}, \beta_{\text {out }}\right)$ if $x_{0} \in \Omega$, and $\left(\alpha_{0}^{*}, \beta_{0}^{*}, \alpha_{1}^{*}, \beta_{1}^{*}\right)=\left(\alpha_{\text {out }}, \beta_{\text {out }}, \alpha_{\text {in }}, \beta_{\text {in }}\right)$ if $x_{0} \in D \backslash \bar{\Omega}$. Hence $\alpha_{1}^{*} \neq \alpha_{0}^{*}$. In connexion with Remark 2.1 we assume that

$$
\frac{\alpha_{1}^{*}}{\alpha_{0}^{*}}=\frac{\beta_{1}^{*}}{\beta_{0}^{*}}
$$

\subsection{A preliminary result}

A proof of the following proposition can be found in [6].

Proposition 3.1. Let $\mathcal{V}$ be a Hilbert space. For all $\varepsilon \in\left[0, \varepsilon_{0}\right), \varepsilon_{0}>0$, consider a vector $u_{\varepsilon} \in \mathcal{V}$ solution of a variational problem of the form

$$
a_{\varepsilon}\left(u_{\varepsilon}, v\right)=\ell_{\varepsilon}(v) \quad \forall v \in \mathcal{V},
$$

where $a_{\varepsilon}$ and $\ell_{\varepsilon}$ are a bilinear form on $\mathcal{V}$ and a linear form on $\mathcal{V}$, respectively. For all $\varepsilon \in\left[0, \varepsilon_{0}\right)$, consider a functional $J_{\varepsilon}: \mathcal{V} \rightarrow \mathbb{R}$ and a linear form $L_{\varepsilon}\left(u_{0}\right) \in \mathcal{V}^{\prime}$. Suppose that the following properties hold.

(1) There exist two numbers $\delta J_{1}$ and $\delta J_{2}$ and a function $\varepsilon \in \mathbb{R}^{+} \mapsto f(\varepsilon) \in \mathbb{R}$ such that, when $\varepsilon$ goes to zero,

$$
\begin{aligned}
& J_{\varepsilon}\left(u_{\varepsilon}\right)=J_{\varepsilon}\left(u_{0}\right)+\left\langle L_{\varepsilon}\left(u_{0}\right), u_{\varepsilon}-u_{0}\right\rangle+f(\varepsilon) \delta J_{1}+o(f(\varepsilon)), \\
& J_{\varepsilon}\left(u_{0}\right)=J_{0}\left(u_{0}\right)+f(\varepsilon) \delta J_{2}+o(f(\varepsilon)) .
\end{aligned}
$$


(2) There exist two numbers $\delta a$ and $\delta \ell$ such that

$$
\begin{aligned}
\left(a_{\varepsilon}-a_{0}\right)\left(u_{0}, v_{\varepsilon}\right) & =f(\varepsilon) \delta a+o(f(\varepsilon)), \\
\left(\ell_{\varepsilon}-\ell_{0}\right)\left(v_{\varepsilon}\right) & =f(\varepsilon) \delta \ell+o(f(\varepsilon)),
\end{aligned}
$$

where $v_{\varepsilon} \in \mathcal{V}$ is an adjoint state satisfying

$$
a_{\varepsilon}\left(\varphi, v_{\varepsilon}\right)=-\left\langle L_{\varepsilon}\left(u_{0}\right), \varphi\right\rangle \quad \forall \varphi \in \mathcal{V}
$$

Then we have

$$
J_{\varepsilon}\left(u_{\varepsilon}\right)-J_{0}\left(u_{0}\right)=f(\varepsilon)\left(\delta a-\delta \ell+\delta J_{1}+\delta J_{2}\right)+o(f(\varepsilon)) .
$$

\section{TOpological SENSItivity ANALYSis OF THE PENALTY FUNCTIONAL}

In this section we check the hypotheses of Proposition 3.1 for the problem under consideration.

\subsection{Adjoint state}

The bilinear and linear forms associated with problem (3.1) are defined in the space $\mathcal{V}=\left\{u \in H^{1}(\Omega)\right.$, $\left.u_{\mid \Gamma_{D}}=0\right\}$ by

$$
\begin{aligned}
a_{\varepsilon}(u, v) & =\int_{D} \alpha_{\varepsilon} \nabla u \cdot \nabla v \mathrm{~d} x \quad \forall u, v \in \mathcal{V} \\
\ell_{\varepsilon}(v) & =\int_{\Gamma_{N}} g v \mathrm{~d} s \quad \forall v \in \mathcal{V}
\end{aligned}
$$

Note that, as above, we will always denote the duality pairing between $H_{00}^{1 / 2}\left(\Gamma_{N}\right)$ and its dual by an integral. Although the penalty functional (3.2) is not Fréchet-differentiable on $\mathcal{V}$ unless $\Phi$ is affine (see [8]), we define its tangent linear approximation at the point $u_{0}$ (unperturbed solution) in a natural way by:

$$
\left\langle L_{\varepsilon}\left(u_{0}\right), \varphi\right\rangle=\int_{\tilde{D}} \beta_{\varepsilon} \Phi^{\prime}\left(\frac{1}{2} B \nabla u_{0} \cdot \nabla u_{0}\right) B \nabla u_{0} \cdot \nabla \varphi \mathrm{d} x \quad \forall \varphi \in \mathcal{V} .
$$

However, to assure that this expression is well-defined, we make the additional assumption that $\Phi^{\prime}$ is bounded. Then we define the function

$$
k_{1}=\Phi^{\prime}\left(\frac{1}{2} B \nabla u_{0} \cdot \nabla u_{0}\right) \chi_{\tilde{D}}
$$

where $\chi_{\tilde{D}}$ is the characteristic function of $\tilde{D}$. We derive from (3.10) that the adjoint state solves:

$$
\left\{\begin{aligned}
-\operatorname{div}\left(\alpha_{\varepsilon} \nabla v_{\varepsilon}\right) & =+\operatorname{div}\left(\beta_{\varepsilon} k_{1} B \nabla u_{0}\right) & \text { in } & D \\
v_{\varepsilon} & =0 & \text { on } & \Gamma_{D} \\
\alpha_{\varepsilon} \partial_{n} v_{\varepsilon} & =-\beta_{\varepsilon} k_{1} B \nabla u_{0} . n & & \text { on } \Gamma_{N} .
\end{aligned}\right.
$$

\subsection{Variation of the bilinear form}

We begin by analyzing the asymptotic behavior of the quantity:

$$
\left(a_{\varepsilon}-a_{0}\right)\left(u_{0}, v_{\varepsilon}\right)=\int_{\omega_{\varepsilon}}\left(\alpha_{1}-\alpha_{0}\right) \nabla u_{0} \cdot \nabla v_{\varepsilon} \mathrm{d} x .
$$


We take $\varepsilon$ sufficiently small so that $\alpha_{1}, \alpha_{0}, \beta_{1}, \beta_{0}$ are constant in $\omega_{\varepsilon}$. By introducing the variation $\tilde{v}_{\varepsilon}=v_{\varepsilon}-v_{0}$, we decompose (4.5) as:

$$
\left(a_{\varepsilon}-a_{0}\right)\left(u_{0}, v_{\varepsilon}\right)=\pi \varepsilon^{2}\left(\alpha_{1}^{*}-\alpha_{0}^{*}\right) \nabla u_{0}\left(x_{0}\right) \cdot \nabla v_{0}\left(x_{0}\right)+\left(\alpha_{1}^{*}-\alpha_{0}^{*}\right) \int_{\omega_{\varepsilon}} \nabla u_{0} \cdot \nabla \tilde{v}_{\varepsilon} \mathrm{d} x+\mathcal{E}_{1}(\varepsilon),
$$

with

$$
\mathcal{E}_{1}(\varepsilon)=\left(\alpha_{1}^{*}-\alpha_{0}^{*}\right) \int_{\omega_{\varepsilon}}\left[\nabla u_{0} \cdot \nabla v_{0}-\nabla u_{0}\left(x_{0}\right) \cdot \nabla v_{0}\left(x_{0}\right)\right] \mathrm{d} x .
$$

Starting from (4.4) and using (3.4), we obtain that $\tilde{v}_{\varepsilon}$ solves:

$$
\left\{\begin{aligned}
-\operatorname{div}\left(\alpha_{\varepsilon} \nabla \tilde{v}_{\varepsilon}\right) & =0 & & \text { in } \omega_{\varepsilon} \cup\left(D \backslash \overline{\omega_{\varepsilon}}\right), \\
{\left[\alpha_{\varepsilon} \partial_{n} \tilde{v}_{\varepsilon}\right] } & =-\left(\alpha_{1}^{*}-\alpha_{0}^{*}\right)\left(\frac{\beta_{0}^{*}}{\alpha_{0}^{*}} k_{1} B \nabla u_{0} \cdot n+\nabla v_{0} \cdot n\right) & & \text { on } \quad \partial \omega_{\varepsilon}, \\
\tilde{v}_{\varepsilon} & =0 & & \text { on } \quad \Gamma_{D}, \\
\partial_{n} \tilde{v}_{\varepsilon} & =0 & & \text { on } \Gamma_{N} .
\end{aligned}\right.
$$

The notation $[f]$ stands for the jump of the function $f$ across $\partial \omega_{\varepsilon}$ with the convention of a positive sign on the interior side. We set

$$
V=\frac{\beta_{0}^{*}}{\alpha_{0}^{*}} k_{1}\left(x_{0}\right) B \nabla u_{0}\left(x_{0}\right)+\nabla v_{0}\left(x_{0}\right),
$$

and we approximate $\tilde{v}_{\varepsilon}$ by the solution $h_{\varepsilon}^{V}$ of the auxiliary problem

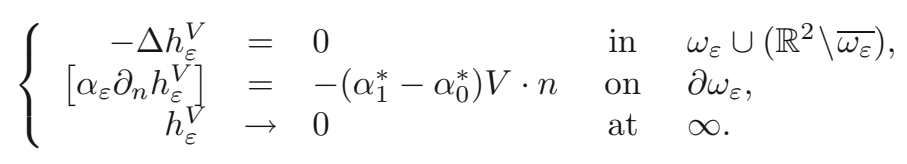

Possibly shifting the coordinate system, we assume from now on for simplicity that $x_{0}=0$. For a circular inclusion, the function $h_{\varepsilon}^{V}$ is known explicitly as:

$$
h_{\varepsilon}^{V}(x)=-\rho \times\left\{\begin{array}{l}
V \cdot x \text { in } \omega_{\varepsilon}, \\
\varepsilon^{2} \frac{V \cdot x}{|x|^{2}} \text { in } \mathbb{R}^{2} \backslash \omega_{\varepsilon},
\end{array}\right.
$$

with

$$
\rho=\frac{\alpha_{1}^{*}-\alpha_{0}^{*}}{\alpha_{1}^{*}+\alpha_{0}^{*}}
$$

It admits the gradient:

$$
\nabla h_{\varepsilon}^{V}(x)=-\rho \times\left\{\begin{array}{l}
V \text { in } \omega_{\varepsilon}, \\
\varepsilon^{2}\left[\frac{V}{|x|^{2}}-2(V . x) \frac{x}{|x|^{4}}\right] \text { in } \mathbb{R}^{2} \backslash \omega_{\varepsilon} .
\end{array}\right.
$$

Denoting by

$$
\begin{aligned}
& \mathcal{E}_{2}(\varepsilon)=\left(\alpha_{1}^{*}-\alpha_{0}^{*}\right) \int_{\omega_{\varepsilon}} \nabla u_{0} \cdot \nabla\left(\tilde{v}_{\varepsilon}-h_{\varepsilon}^{V}\right) \mathrm{d} x, \\
& \mathcal{E}_{3}(\varepsilon)=\left(\alpha_{1}^{*}-\alpha_{0}^{*}\right) \int_{\omega_{\varepsilon}}\left(\nabla u_{0}-\nabla u_{0}\left(x_{0}\right)\right) \cdot \nabla h_{\varepsilon}^{V} \mathrm{~d} x,
\end{aligned}
$$


we obtain straightforwardly

$$
\begin{aligned}
\left(\alpha_{1}^{*}-\alpha_{0}^{*}\right) \int_{\omega_{\varepsilon}} \nabla u_{0} \cdot \nabla \tilde{v}_{\varepsilon} \mathrm{d} x & =\left(\alpha_{1}^{*}-\alpha_{0}^{*}\right) \int_{\omega_{\varepsilon}} \nabla u_{0}\left(x_{0}\right) \cdot \nabla h_{\varepsilon}^{V} \mathrm{~d} x+\mathcal{E}_{2}(\varepsilon)+\mathcal{E}_{3}(\varepsilon) \\
& =-\pi \varepsilon^{2}\left(\alpha_{1}^{*}-\alpha_{0}^{*}\right) \rho \nabla u_{0}\left(x_{0}\right) \cdot V+\mathcal{E}_{2}(\varepsilon)+\mathcal{E}_{3}(\varepsilon) .
\end{aligned}
$$

After rearrangement, we arrive at:

$$
\left(a_{\varepsilon}-a_{0}\right)\left(u_{0}, v_{\varepsilon}\right)=\pi \varepsilon^{2} \rho\left[2 \alpha_{0}^{*} \nabla u_{0}\left(x_{0}\right) \cdot \nabla v_{0}\left(x_{0}\right)-\left(\beta_{1}^{*}-\beta_{0}^{*}\right) k_{1}\left(x_{0}\right) B \nabla u_{0}\left(x_{0}\right) \cdot \nabla u_{0}\left(x_{0}\right)\right]+\sum_{i=1}^{3} \mathcal{E}_{i}(\varepsilon) .
$$

We shall prove in Section 5 that $\mathcal{E}_{i}(\varepsilon)=o\left(\varepsilon^{2}\right)$ for $i=1,2,3$. We conclude that (3.8) holds true with

$$
\begin{gathered}
f(\varepsilon)=\varepsilon^{2} \\
\delta a=\pi \rho\left[2 \alpha_{0}^{*} \nabla u_{0}\left(x_{0}\right) \cdot \nabla v_{0}\left(x_{0}\right)-\left(\beta_{1}^{*}-\beta_{0}^{*}\right) k_{1}\left(x_{0}\right) B \nabla u_{0}\left(x_{0}\right) \cdot \nabla u_{0}\left(x_{0}\right)\right] .
\end{gathered}
$$

\subsection{Variation of the linear form}

In our case, $\ell_{\varepsilon}$ is independent of $\varepsilon$, hence

$$
\delta \ell=0 .
$$

\subsection{Partial variation of the penalty functional with respect to the state}

We have to study the variation

$$
\begin{aligned}
V_{J 1}(\varepsilon) & :=J_{\varepsilon}\left(u_{\varepsilon}\right)-J_{\varepsilon}\left(u_{0}\right)-\left\langle L_{\varepsilon}\left(u_{0}\right), u_{\varepsilon}-u_{0}\right\rangle \\
& =\int_{\tilde{D}} \beta_{\varepsilon}\left[\Phi\left(\frac{1}{2} B \nabla u_{\varepsilon} \cdot \nabla u_{\varepsilon}\right)-\Phi\left(\frac{1}{2} B \nabla u_{0} \cdot \nabla u_{0}\right)-\Phi^{\prime}\left(\frac{1}{2} B \nabla u_{0} \cdot \nabla u_{0}\right) B \nabla u_{0} \cdot \nabla\left(u_{\varepsilon}-u_{0}\right)\right] \mathrm{d} x .
\end{aligned}
$$

Setting $\tilde{u}_{\varepsilon}=u_{\varepsilon}-u_{0}$, we obtain

$$
\begin{array}{r}
V_{J 1}(\varepsilon)=\int_{\tilde{D}} \beta_{\varepsilon}\left[\Phi\left(\frac{1}{2} B \nabla u_{0} \cdot \nabla u_{0}+B \nabla u_{0} \cdot \nabla \tilde{u}_{\varepsilon}+\frac{1}{2} B \nabla \tilde{u}_{\varepsilon} \cdot \nabla \tilde{u}_{\varepsilon}\right)-\Phi\left(\frac{1}{2} B \nabla u_{0} \cdot \nabla u_{0}\right)\right. \\
\left.-\Phi^{\prime}\left(\frac{1}{2} B \nabla u_{0} \cdot \nabla u_{0}\right) B \nabla u_{0} \cdot \nabla \tilde{u}_{\varepsilon}\right] \mathrm{d} x .
\end{array}
$$

By difference, we find that $\tilde{u}_{\varepsilon}$ solves:

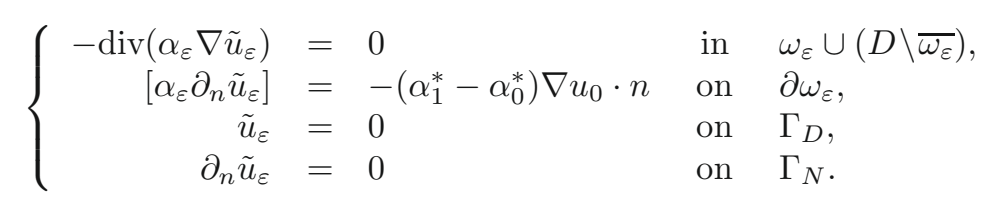

Setting now $U=\nabla u_{0}\left(x_{0}\right)$, we approximate $\tilde{u}_{\varepsilon}$ by $h_{\varepsilon}^{U}$ solution to (4.7) with $U$ substituted for $V$. It comes:

$$
\begin{array}{r}
V_{J 1}(\varepsilon)=\int_{\tilde{D}} \beta_{\varepsilon}\left[\Phi\left(\frac{1}{2} B \nabla u_{0} \cdot \nabla u_{0}+B \nabla u_{0} \cdot \nabla h_{\varepsilon}^{U}+\frac{1}{2} B \nabla h_{\varepsilon}^{U} \cdot \nabla h_{\varepsilon}^{U}\right)-\Phi\left(\frac{1}{2} B \nabla u_{0} \cdot \nabla u_{0}\right)\right. \\
\left.-\Phi^{\prime}\left(\frac{1}{2} B \nabla u_{0} \cdot \nabla u_{0}\right) B \nabla u_{0} \cdot \nabla h_{\varepsilon}^{U}\right] \mathrm{d} x+\mathcal{E}_{4}(\varepsilon),
\end{array}
$$


with

$$
\begin{aligned}
\mathcal{E}_{4}(\varepsilon)= & \int_{\tilde{D}} \beta_{\varepsilon}\left[\Phi\left(\frac{1}{2} B \nabla u_{0} \cdot \nabla u_{0}+B \nabla u_{0} \cdot \nabla \tilde{u}_{\varepsilon}+\frac{1}{2} B \nabla \tilde{u}_{\varepsilon} \cdot \nabla \tilde{u}_{\varepsilon}\right)\right. \\
& \left.-\Phi\left(\frac{1}{2} B \nabla u_{0} \cdot \nabla u_{0}+B \nabla u_{0} \cdot \nabla h_{\varepsilon}^{U}+\frac{1}{2} B \nabla h_{\varepsilon}^{U} \cdot \nabla h_{\varepsilon}^{U}\right)-\Phi^{\prime}\left(\frac{1}{2} B \nabla u_{0} \cdot \nabla u_{0}\right) B \nabla u_{0} \cdot\left(\nabla \tilde{u}_{\varepsilon}-\nabla h_{\varepsilon}^{U}\right)\right] \mathrm{d} x .
\end{aligned}
$$

We assume that $x_{0} \in \tilde{D}$. Otherwise, when $x_{0} \in D \backslash \overline{\tilde{D}}$, the theory developed in [6] applies and provides $\delta J_{1}=0$. We exclude of our study the special case where $x_{0} \in \partial \tilde{D}$. In view of the decay of $\nabla h_{\varepsilon}^{U}$ as well as the regularity of $u_{0}$ near $x_{0}$, we write

$$
\begin{aligned}
V_{J 1}(\varepsilon)=\int_{\mathbb{R}^{2}} \beta_{\varepsilon}^{*}\left[\Phi\left(\frac{1}{2} B U \cdot U+B U \cdot \nabla h_{\varepsilon}^{U}+\frac{1}{2} B \nabla h_{\varepsilon}^{U} \cdot \nabla h_{\varepsilon}^{U}\right)\right. & -\Phi\left(\frac{1}{2} B U \cdot U\right) \\
& \left.-\Phi^{\prime}\left(\frac{1}{2} B U \cdot U\right) B U \cdot \nabla h_{\varepsilon}^{U}\right] \mathrm{d} x+\mathcal{E}_{4}(\varepsilon)+\mathcal{E}_{5}(\varepsilon),
\end{aligned}
$$

with the adequate remainder $\mathcal{E}_{5}(\varepsilon)$ and $\beta_{\varepsilon}^{*}(x)=\beta_{1}^{*}$ if $x \in \omega_{\varepsilon}, \beta_{\varepsilon}^{*}(x)=\beta_{0}^{*}$ otherwise. Next we make the splitting

$$
V_{J 1}(\varepsilon)=V_{J 11}(\varepsilon)+V_{J 12}(\varepsilon)+\mathcal{E}_{4}(\varepsilon)+\mathcal{E}_{5}(\varepsilon)
$$

where $V_{J 11}$ and $V_{J 12}$ correspond to an integration over $\omega_{\varepsilon}$ and $\mathbb{R}^{2} \backslash \overline{\omega_{\varepsilon}}$, respectively. Using (4.10) we obtain that

$$
V_{J 11}(\varepsilon)=\varepsilon^{2} \pi \beta_{1}^{*}\left[\Phi\left((1-\rho)^{2} \frac{1}{2} B U \cdot U\right)-\Phi\left(\frac{1}{2} B U \cdot U\right)+\rho \Phi^{\prime}\left(\frac{1}{2} B U \cdot U\right) B U \cdot U\right] .
$$

Next, we define the function independent of $\varepsilon$

$$
H_{\rho}^{U}(x)=\nabla h_{\varepsilon}^{U}(\varepsilon x)=-\rho\left[\frac{U}{|x|^{2}}-2(U \cdot x) \frac{x}{|x|^{4}}\right] \quad \forall x \in \mathbb{R}^{2} \backslash \bar{\omega} .
$$

A change of variable yields

$$
V_{J 12}(\varepsilon)=\varepsilon^{2} \int_{\mathbb{R}^{2} \backslash \bar{\omega}} \beta_{0}^{*}\left[\Phi\left(\frac{1}{2} B U \cdot U+B U \cdot H_{\rho}^{U}+\frac{1}{2} B H_{\rho}^{U} \cdot H_{\rho}^{U}\right)-\Phi\left(\frac{1}{2} B U \cdot U\right)-\Phi^{\prime}\left(\frac{1}{2} B U \cdot U\right) B U \cdot H_{\rho}^{U}\right] \mathrm{d} x .
$$

We set

$$
\Psi_{\rho}(U)=\int_{\mathbb{R}^{2} \backslash \bar{\omega}}\left[\Phi\left(\frac{1}{2} B\left(U+H_{\rho}^{U}\right) \cdot\left(U+H_{\rho}^{U}\right)\right)-\Phi\left(\frac{1}{2} B U \cdot U\right)-\Phi^{\prime}\left(\frac{1}{2} B U \cdot U\right)\left(B U \cdot H_{\rho}^{U}+\frac{1}{2} B H_{\rho}^{U} \cdot H_{\rho}^{U}\right)\right] \mathrm{d} x .
$$

The extra term $\frac{1}{2} B H_{\rho}^{U} \cdot H_{\rho}^{U}$ has been added so that $\Psi_{\rho}(U)$ vanishes whenever $\Phi$ is linear. Thus we have

$$
V_{J 12}(\varepsilon)=\varepsilon^{2} \beta_{0}^{*}\left[\Psi_{\rho}(U)+\frac{1}{2} \Phi^{\prime}\left(\frac{1}{2} B U \cdot U\right) \int_{\mathbb{R}^{2} \backslash \bar{\omega}} B H_{\rho}^{U} \cdot H_{\rho}^{U} \mathrm{~d} x\right] .
$$

A symbolic calculus of the above integral provides

$$
V_{J 12}(\varepsilon)=\varepsilon^{2} \beta_{0}^{*}\left[\Psi_{\rho}(U)+\rho^{2} \frac{\pi}{4} k_{1}\left(x_{0}\right) \operatorname{tr}(B)|U|^{2}\right],
$$


where $\operatorname{tr}(B)$ stands for the trace of the matrix $B$. We shall prove in Section 5 that $\mathcal{E}_{4}(\varepsilon)+\mathcal{E}_{5}(\varepsilon)=o\left(\varepsilon^{2}\right)$. Therefore we get

$$
\delta J_{1}=\pi \beta_{1}^{*}\left[\Phi\left((1-\rho)^{2} \frac{1}{2} B U \cdot U\right)-\Phi\left(\frac{1}{2} B U \cdot U\right)+\rho k_{1}\left(x_{0}\right) B U \cdot U\right]+\beta_{0}^{*}\left[\Psi_{\rho}(U)+\rho^{2} \frac{\pi}{4} k_{1}\left(x_{0}\right) \operatorname{tr}(B)|U|^{2}\right] .
$$

\subsection{Partial variation of the penalty functional with respect to the domain}

We assume again that $x_{0} \notin \partial \tilde{D}$. We have

$$
\begin{aligned}
V J_{2}(\varepsilon) & :=J_{\varepsilon}\left(u_{0}\right)-J_{0}\left(u_{0}\right) \\
& =\int_{\omega_{\varepsilon} \cap \tilde{D}}\left(\beta_{1}-\beta_{0}\right) \Phi\left(\frac{1}{2} B \nabla u_{0} \cdot \nabla u_{0}\right) \mathrm{d} x \\
& =\pi \varepsilon^{2}\left(\beta_{1}^{*}-\beta_{0}^{*}\right) \chi_{\tilde{D}}\left(x_{0}\right) \Phi\left(\frac{1}{2} B \nabla u_{0}\left(x_{0}\right) \cdot \nabla u_{0}\left(x_{0}\right)\right)+\mathcal{E}_{6}(\varepsilon),
\end{aligned}
$$

with $\mathcal{E}_{6}(\varepsilon)=o\left(\varepsilon^{2}\right)($ see Sect. 5). Thus we obtain

$$
\delta J_{2}=\pi\left(\beta_{1}^{*}-\beta_{0}^{*}\right) \chi_{\tilde{D}}\left(x_{0}\right) \Phi\left(\frac{1}{2} B \nabla u_{0}\left(x_{0}\right) \cdot \nabla u_{0}\left(x_{0}\right)\right)
$$

\subsection{Topological derivative}

Before stating the main result of this paper, we make a regularity assumption which will be useful to establish the needed estimates.

Assumption 4.1. If $x_{0} \in \tilde{D}$, then there exists $\eta>0$ such that:

(1) $B\left(x_{0}, 2 \eta\right) \subset \tilde{D}$

(2) $u_{0}, v_{0} \in \mathcal{C}^{1, \alpha}\left(\overline{B\left(x_{0}, 2 \eta\right)}\right), \alpha>0$

(3) $u_{0} \in W^{1,4}\left(\tilde{D} \backslash \overline{B\left(x_{0}, 2 \eta\right)}\right)$;

(4) every function $u \in H^{1}\left(D \backslash \overline{B\left(x_{0}, \eta\right)}\right)$ satisfying

$$
\left\{\begin{array}{rllll}
-\operatorname{div}\left(\alpha_{0} \nabla u\right) & = & 0 & \text { in } & D \backslash \overline{B\left(x_{0}, \eta\right)} \\
u & = & 0 & \text { on } & \Gamma_{D} \\
\alpha_{0} \partial_{n} u & = & 0 & \text { on } & \Gamma_{N}
\end{array}\right.
$$

belongs to $W^{1,4}\left(\tilde{D} \backslash \overline{B\left(x_{0}, 2 \eta\right)}\right)$.

Remark 4.2. Assumption 4.1 is little restrictive. It is satisfied for instance in the following situation, which corresponds to the problems studied in Section 8:

- $\Phi^{\prime \prime}$, calculated in the sense of distributions, belongs to $L_{\text {loc }}^{\infty}\left(\mathbb{R}_{+}\right)$, which implies that $k_{1}$ is in $W^{1, \infty}$ in a neighborhood of $x_{0}$ and thus guarantees by elliptic regularity that $v_{0}$ is locally $W^{2, p}$ for any $p>2$, itself imbedded in $\mathcal{C}^{1,1-2 / p}$ (see e.g. [17]);

- $D$ is a Lipschitz polygon with edges $\left(\Gamma_{j}\right)_{j=1, \ldots, N}$ and vertices $\left(S_{i}\right)_{i=1, \ldots, P}$; 
- on each edge $\Gamma_{j}$ the boundary condition is either $u=0$ or $\partial_{n} u=g_{j} \in H^{1 / 2+\delta}\left(\Gamma_{j}\right), \delta>0$;

- if a vertex $S_{i} \in \Gamma_{j} \cap \Gamma_{j+1}$ where the type of boundary condition changes belongs to $\partial \tilde{D}$, then the interior angle between $\Gamma_{j}$ and $\Gamma_{j+1}$ is lower than $\pi$ and, in case it is equal to $\frac{\pi}{2}$, the two boundary conditions are compatible (see [18], Cor. 4.4.3.8);

- the interface $\overline{\partial \Omega \backslash \partial D}$ is the disjoint union of $\operatorname{arcs}\left(\mathcal{I}_{j}\right)_{j=1, \ldots, K}$ of class $\mathcal{C}^{1,1}$ such that each arc touches the external boundary $\partial D$ in two distinct points denoted by $\mathcal{I}_{j} \cap \partial D=\left\{P_{j 1}, P_{j 2}\right\}$;

- if a junction point $P_{j k}$ coincides with a vertex $S_{i}$ and belongs to $\partial \tilde{D}$, then the two interior angles defined by these curves are less than or equal to $\pi$ and the boundary condition is locally either $u=0$ or $\partial_{n} u=0$ without changing.

This property is obtained by collecting results from $[18,27,30]$ and using Sobolev imbedding theorems (see e.g. [1]). We refer to these documents for possible extensions.

In view of Proposition 3.1, gathering (4.11), (4.12), (4.13), (4.19) and (4.20) provides the following result.

Theorem 4.3. Consider a function $\Phi \in \mathcal{C}^{1}\left(\mathbb{R}_{+} ; \mathbb{R}\right)$ with $\Phi^{\prime}$ Lipschitz continuous and bounded, and choose $x_{0} \in D \backslash \partial \Omega \backslash \partial \tilde{D}$. We assume that all the assumptions of Section 3.1 are satisfied as well as Assumption 4.1. Then the reduced penalty function $j(\varepsilon)=J_{\varepsilon}\left(u_{\varepsilon}\right)$ admits the asymptotic expansion

$$
j(\varepsilon)-j(0)=\varepsilon^{2} G\left(x_{0}\right)+o\left(\varepsilon^{2}\right),
$$

where the topological derivative (also called topological gradient) $G$ is given by:

$$
\begin{aligned}
G=\pi \rho\left[2 \alpha_{0} \nabla u_{0} \cdot \nabla v_{0}+\beta_{0} k_{1} B \nabla\right. & \left.u_{0} \cdot \nabla u_{0}\right]+\pi \beta_{1} \chi_{\tilde{D}} \Phi\left((1-\rho)^{2} \frac{1}{2} B \nabla u_{0} \cdot \nabla u_{0}\right) \\
& +\beta_{0} \chi_{\tilde{D}}\left[\Psi_{\rho}\left(\nabla u_{0}\right)+\rho^{2} \frac{\pi}{4} k_{1} \operatorname{tr}(B)\left|\nabla u_{0}\right|^{2}\right]-\pi \beta_{0} \chi_{\tilde{D}} \Phi\left(\frac{1}{2} B \nabla u_{0} \cdot \nabla u_{0}\right) .
\end{aligned}
$$

We recall that $k_{1}, \rho$ and $\Psi_{\rho}$ are defined by (4.3), (4.9) and (4.18), respectively. The adjoint state $v_{0}$ is the unique solution of the boundary value problem:

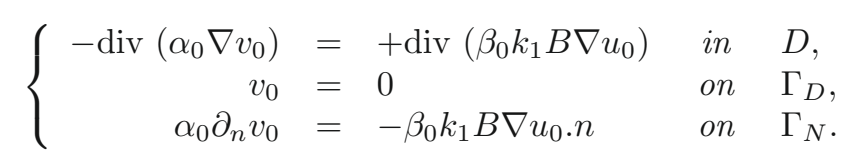

\section{Remark 4.4.}

(1) The assumption of boundedness of $\Phi^{\prime}$, which implies the Lipschitz continuity of $\Phi$, will be used several times in the proof of Theorem 4.3, but it can be weakened. However, in order to be assured of the existence of an $H^{1}$ solution to (4.4) when $\nabla u_{0} \in L^{4}(D)$ (which is the best $L^{p}$ regularity to be expected in a Lipschitz domain), we need a growth condition of the type $\left|\Phi^{\prime}(x)\right| \leq a+b \sqrt{x}$ (see [8]). This condition is obviously not fulfilled by penalty functions with quadratic growth. Conversely, if $\Phi^{\prime}$ had a linear growth, we would need $\nabla u_{0} \in L^{6}(D)$, hence every angle would have to be less that $3 \pi / 2$. Thus we have assumed that $\Phi^{\prime}$ is bounded for simplicity of the proofs as well as for practical reasons.

(2) Expression (4.22) is related to a very general result, therefore not fully explicit, stated in [25] for an arbitrarily-shaped perforation with Neumann condition on its boundary. However, the proof given in [25] is valid only if $D$ has a smooth boundary. The capability to deal with domains with corners is essential here, and significantly complicates the analysis.

(3) When $\Phi$ is convex, the function $\Psi_{\rho}$ is everywhere nonnegative by construction. 


\subsection{Expression of $\Psi_{\rho}(U)$ in the isotropic case}

When $B$ is the identity matrix, we observe that $\Psi_{\rho}(U)$ is invariant by rotation of the vector $U$. Therefore we can write

$$
\Psi_{\rho}(U)=\hat{\Psi}_{\rho}\left(\frac{1}{2}|U|^{2}\right)
$$

Plugging (4.17) into (4.18) and taking $U$ parallel to the $x$-axis yields after a change of variable and rearrangement:

$$
\hat{\Psi}_{\rho}(\sigma)=\rho \int_{0}^{\rho} \int_{0}^{\pi} \frac{1}{t^{2}}\left[\Phi\left(\sigma\left(1+t^{2}+2 t \cos \theta\right)\right)-\Phi(\sigma)-\Phi^{\prime}(\sigma) \sigma\left(t^{2}+2 t \cos \theta\right)\right] \mathrm{d} \theta \mathrm{d} t .
$$

\section{Auxiliary estimates}

\subsection{Preliminary lemmas}

Lemma 5.1. (1) For any vector $V \in \mathbb{R}^{2}$ and any positive radius $R$, we have

$$
\begin{gathered}
\left\|h_{\varepsilon}^{V}\right\|_{L^{2}(D)}=O\left(\varepsilon^{3 / 2}\right), \\
\left\|\nabla h_{\varepsilon}^{V}\right\|_{L^{p}(D)}=O\left(\varepsilon^{2 / p}\right) \quad \forall p>1, \\
\left\|h_{\varepsilon}^{V}\right\|_{L^{p}\left(D \backslash B\left(x_{0}, R\right)\right)}+\left\|\nabla h_{\varepsilon}^{V}\right\|_{L^{p}\left(D \backslash B\left(x_{0}, R\right)\right)}=O\left(\varepsilon^{2}\right) \quad \forall p \geq 1 .
\end{gathered}
$$

(2) Given a function $\psi: D \rightarrow \mathbb{R}^{2}$ which is Hölder continuous in the vicinity of $x_{0}$, consider the solution $w_{\varepsilon}$ of the system:

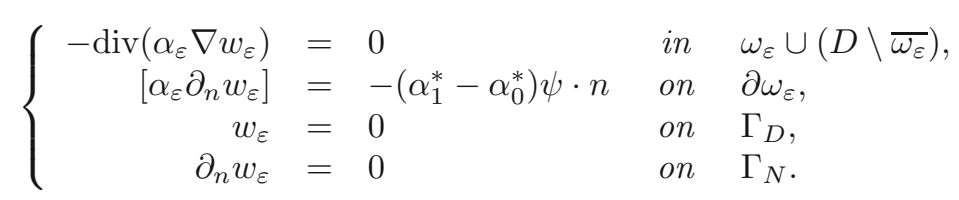

Then we have

$$
\left\|w_{\varepsilon}-h_{\varepsilon}^{\psi\left(x_{0}\right)}\right\|_{H^{1}(D)}=o(\varepsilon) .
$$

Proof. The first part of the lemma derives straightforwardly from the expressions (4.8) and (4.10). Therefore we concentrate on the second part. To do so, we take an arbitrary test function $\varphi \in \mathcal{V}$. On the one hand, the variational formulation associated with (5.1) provides

$$
\int_{D} \alpha_{\varepsilon} \nabla w_{\varepsilon} \cdot \nabla \varphi \mathrm{d} x=-\left(\alpha_{1}^{*}-\alpha_{0}^{*}\right) \int_{\partial \omega_{\varepsilon}}(\psi \cdot n) \varphi \mathrm{d} s
$$

On the other hand, the Green formula together with (4.7) yield

$$
\int_{D} \alpha_{\varepsilon} \nabla h_{\varepsilon} \cdot \nabla \varphi \mathrm{d} x=-\left(\alpha_{1}^{*}-\alpha_{0}^{*}\right) \int_{\partial \omega_{\varepsilon}}\left(\psi\left(x_{0}\right) \cdot n\right) \varphi \mathrm{d} s+\left(\alpha_{\text {in }}-\alpha_{\text {out }}\right) \int_{\partial \Omega \cap D} \partial_{n} h_{\varepsilon} \varphi \mathrm{d} s+\int_{\Gamma_{N}} \alpha_{0} \partial_{n} h_{\varepsilon} \varphi \mathrm{d} s .
$$

For notational simplicity, we have dropped the superscript in $h_{\varepsilon}^{\psi\left(x_{0}\right)}$. It follows that the difference $e_{\varepsilon}:=w_{\varepsilon}-h_{\varepsilon}$ satisfies

$$
\int_{D} \alpha_{\varepsilon} \nabla e_{\varepsilon} \cdot \nabla \varphi \mathrm{d} x=-\left(\alpha_{1}^{*}-\alpha_{0}^{*}\right) \int_{\partial \omega_{\varepsilon}}\left(\psi-\psi\left(x_{0}\right)\right) \cdot n \varphi \mathrm{d} s-\left(\alpha_{\text {in }}-\alpha_{\text {out }}\right) \int_{\partial \Omega \cap D} \partial_{n} h_{\varepsilon} \varphi \mathrm{d} s-\int_{\Gamma_{N}} \alpha_{0} \partial_{n} h_{\varepsilon} \varphi \mathrm{d} s
$$


By a change of variable, the $\alpha$-Hölder continuity of $\psi$ in the vicinity of $x_{0}(0<\alpha<1)$ and the trace theorem, we get for $\varepsilon$ small enough

$$
\begin{aligned}
\left|\int_{\partial \omega_{\varepsilon}}\left(\psi-\psi\left(x_{0}\right)\right) \cdot n \varphi \mathrm{d} s\right| & =\varepsilon\left|\int_{\partial \omega}\left(\psi(\varepsilon x)-\psi\left(x_{0}\right)\right) \cdot n \varphi(\varepsilon x) \mathrm{d} s\right| \\
& \leq c \varepsilon^{1+\alpha}\|\varphi(\varepsilon x)\|_{H^{1 / 2}(\partial \omega)} \\
& \leq c \varepsilon^{1+\alpha}\|\varphi(\varepsilon x)\|_{H^{1}(\omega)} \\
& \leq c \varepsilon^{\alpha}\|\varphi\|_{L^{2}\left(\omega_{\varepsilon}\right)}+c \varepsilon^{1+\alpha}\|\nabla \varphi\|_{L^{2}\left(\omega_{\varepsilon}\right)} .
\end{aligned}
$$

The Hölder inequality and the Sobolev imbedding theorem yield $\|\varphi\|_{L^{2}\left(\omega_{\varepsilon}\right)} \leq c \varepsilon^{1 / p}\|\varphi\|_{L^{2 p /(p-1)}\left(\omega_{\varepsilon}\right)} \leq c \varepsilon^{1 / p}$ $\|\varphi\|_{H^{1}(D)}$ for any $p>1$. It comes

$$
\left|\int_{\partial \omega_{\varepsilon}}\left(\psi-\psi\left(x_{0}\right)\right) \cdot n \varphi \mathrm{d} s\right| \leq c\left(\varepsilon^{\alpha+1 / p}+\varepsilon^{\alpha+1}\right)\|\varphi\|_{H^{1}(D)} .
$$

We estimate the other terms of (5.2) with the help of the first part of the lemma. We arrive at

$$
\left|\int_{D} \alpha_{\varepsilon} \nabla e_{\varepsilon} \cdot \nabla \varphi \mathrm{d} x\right| \leq c\left(\varepsilon^{\alpha+1 / p}+\varepsilon^{\alpha+1}+\varepsilon^{2}\right)\|\varphi\|_{H^{1}(D)} .
$$

Besides, we have the boundary condition $e_{\varepsilon}=-h_{\varepsilon}$ on $\Gamma_{D}$, which satisfies $\left\|h_{\varepsilon}\right\|_{H^{1 / 2}\left(\Gamma_{D}\right)} \leq c \varepsilon^{2}$. Thus we obtain by elliptic regularity

$$
\left\|e_{\varepsilon}\right\|_{H^{1}(D)} \leq c\left(\varepsilon^{\alpha+1 / p}+\varepsilon^{\alpha+1}+\varepsilon^{2}\right) .
$$

The proof is achieved by choosing any $p \in\left(1, \frac{1}{1-\alpha}\right)$.

Lemma 5.2. For the variation $\tilde{u}_{\varepsilon}=u_{\varepsilon}-u_{0}$ we have

$$
\begin{gathered}
\left\|\tilde{u}_{\varepsilon}\right\|_{H^{1}(D)}=O(\varepsilon), \\
\left\|\tilde{u}_{\varepsilon}\right\|_{W^{1,4}\left(\tilde{D} \backslash \overline{\left.B\left(x_{0}, 2 \eta\right)\right)}\right.}=o(\varepsilon) .
\end{gathered}
$$

Proof. (1) In view of (4.15), (5.3) is a straightforward application of Lemma 5.1. Indeed, we have

$$
\left\|\tilde{u}_{\varepsilon}\right\|_{H^{1}(D)} \leq\left\|\tilde{u}_{\varepsilon}-h_{\varepsilon}^{U}\right\|_{H^{1}(D)}+\left\|h_{\varepsilon}^{U}\right\|_{H^{1}(D)} .
$$

The first term is a $o(\varepsilon)$ and the second one is a $O(\varepsilon)$.

(2) Let $\mathcal{B}$ be the set of all functions $u \in H^{1}\left(D \backslash \overline{B\left(x_{0}, \eta\right)}\right)$ satisfying (4.21). Due to Assumption 4.1, we have $\mathcal{B}_{\mid \tilde{D} \backslash \overline{B\left(x_{0}, 2 \eta\right)}} \subset W^{1,4}\left(\tilde{D} \backslash \overline{B\left(x_{0}, 2 \eta\right)}\right)$. It is then easy to check that $\mathcal{B}$ is a Banach space when it is endowed with the norms

$$
\begin{gathered}
\|u\|_{1}=\|u\|_{H^{1}\left(D \backslash \overline{B\left(x_{0}, \eta\right)}\right)}, \\
\|u\|_{2}=\|u\|_{H^{1}\left(D \backslash \overline{B\left(x_{0}, \eta\right)}\right)}+\|u\|_{W^{1,4}\left(\tilde{D} \backslash \overline{B\left(x_{0}, 2 \eta\right)}\right)}
\end{gathered}
$$

By virtue of the open mapping theorem, these norms are equivalent. Using Lemma 5.1 completes the proof. 


\subsection{Asymptotic behavior of the remainders}

We shall prove that $\mathcal{E}_{i}(\varepsilon)=o\left(\varepsilon^{2}\right)$ for $i=1, \ldots, 6$. For simplicity, we use the letter $c$ to denote any constant independent of $\varepsilon$. We focus on the case where $x_{0} \in \tilde{D}$, the other case being treated in [6]. It will appear that the function $\Phi$ needs to be extended to the whole real line. We still denote by $\Phi$ such an extension, constructed in such a way that it is of class $\mathcal{C}^{1}$ on $\mathbb{R}$ with Lipschitz continuous and bounded derivative.

(1) Using the regularity of $u_{0}$ and $v_{0}$ near $x_{0}$, it is straightforward to check that $\mathcal{E}_{1}(\varepsilon)=O\left(\varepsilon^{2+\alpha}\right)$.

(2) It stems from the Cauchy-Schwarz inequality that

$$
\left|\mathcal{E}_{2}(\varepsilon)\right| \leq c \varepsilon\left\|\tilde{v}_{\varepsilon}-h_{\varepsilon}^{V}\right\|_{H^{1}(D)} .
$$

We recall that $V$ is defined by (4.6). Applying Lemma 5.1 with $\psi=\frac{\beta_{0}^{*}}{\beta_{1}^{*}} k_{1} B \nabla u_{0}+\nabla v_{0}$ results in

$$
\left\|\tilde{v}_{\varepsilon}-h_{\varepsilon}^{V}\right\|_{H^{1}(D)}=o(\varepsilon)
$$

We conclude that $\mathcal{E}_{2}(\varepsilon)=o\left(\varepsilon^{2}\right)$.

(3) Using the Cauchy-Schwarz inequality, Lemma 5.1 and the regularity of $u_{0}$ in the vicinity of $x_{0}$, we obtain straightforwardly that $\mathcal{E}_{3}(\varepsilon)=O\left(\varepsilon^{2+\alpha}\right)$.

(4) We use the simplified notations $D_{1}=B\left(x_{0}, 2 \eta\right)$ and $D_{2}=\tilde{D} \backslash \overline{B\left(x_{0}, 2 \eta\right)}$. We make the splitting $\mathcal{E}_{4}(\varepsilon)=\mathcal{E}_{4}^{1}(\varepsilon)+\mathcal{E}_{4}^{21}(\varepsilon)+\mathcal{E}_{4}^{22}(\varepsilon)$ with

$$
\begin{aligned}
\mathcal{E}_{4}^{1}(\varepsilon)= & \int_{\tilde{D}} \beta_{\varepsilon}\left[\Phi\left(\frac{1}{2} B \nabla u_{0} \cdot \nabla u_{0}+B \nabla u_{0} \cdot \nabla \tilde{u}_{\varepsilon}+\frac{1}{2} B \nabla \tilde{u}_{\varepsilon} \cdot \nabla \tilde{u}_{\varepsilon}\right)\right. \\
& \left.-\Phi\left(\frac{1}{2} B \nabla u_{0} \cdot \nabla u_{0}+B \nabla u_{0} \cdot \nabla \tilde{u}_{\varepsilon}+\frac{1}{2} B \nabla h_{\varepsilon}^{U} \cdot \nabla h_{\varepsilon}^{U}\right)\right] \mathrm{d} x, \\
\mathcal{E}_{4}^{2 i}(\varepsilon)= & \int_{D_{i}} \beta_{\varepsilon}\left[\Phi\left(\frac{1}{2} B \nabla u_{0} \cdot \nabla u_{0}+B \nabla u_{0} \cdot \nabla \tilde{u}_{\varepsilon}+\frac{1}{2} B \nabla h_{\varepsilon}^{U} \cdot \nabla h_{\varepsilon}^{U}\right)\right. \\
& \left.-\Phi\left(\frac{1}{2} B \nabla u_{0} \cdot \nabla u_{0}+B \nabla u_{0} \cdot \nabla h_{\varepsilon}^{U}+\frac{1}{2} B \nabla h_{\varepsilon}^{U} \cdot \nabla h_{\varepsilon}^{U}\right)-\Phi^{\prime}\left(\frac{1}{2} B \nabla u_{0} \cdot \nabla u_{0}\right) B \nabla u_{0} \cdot\left(\nabla \tilde{u}_{\varepsilon}-\nabla h_{\varepsilon}^{U}\right)\right] \mathrm{d} x .
\end{aligned}
$$

Using that $\Phi$ is Lipschitz continuous yields

$$
\begin{aligned}
\left|\mathcal{E}_{4}^{1}(\varepsilon)\right| & \leq c \int_{D} \beta_{\varepsilon}\left|\frac{1}{2} B \nabla \tilde{u}_{\varepsilon} \cdot \nabla \tilde{u}_{\varepsilon}-\frac{1}{2} B \nabla h_{\varepsilon}^{U} \cdot \nabla h_{\varepsilon}^{U}\right| \mathrm{d} x \\
& \leq c\left\|\nabla \tilde{u}_{\varepsilon}-\nabla h_{\varepsilon}^{U}\right\|_{L^{2}(D)}\left\|\nabla \tilde{u}_{\varepsilon}+\nabla h_{\varepsilon}^{U}\right\|_{L^{2}(D)} \\
& \leq c\left\|\tilde{u}_{\varepsilon}-h_{\varepsilon}^{U}\right\|_{H^{1}(D)}\left(\left\|\tilde{u}_{\varepsilon}\right\|_{H^{1}(D)}+\left\|h_{\varepsilon}^{U}\right\|_{H^{1}(D)}\right) .
\end{aligned}
$$

Lemmas 5.1 and 5.2 entail $\mathcal{E}_{4}^{1}(\varepsilon)=o\left(\varepsilon^{2}\right)$.

By the mean value theorem, there exists a function $\xi: D_{1} \rightarrow \mathbb{R}$ such that almost everywhere in $D_{1}$

$$
\begin{aligned}
\Phi\left(\frac{1}{2} B \nabla u_{0} \cdot \nabla u_{0}+B \nabla u_{0} \cdot \nabla \tilde{u}_{\varepsilon}+\frac{1}{2} B \nabla h_{\varepsilon}^{U} \cdot \nabla h_{\varepsilon}^{U}\right) \\
-\Phi\left(\frac{1}{2} B \nabla u_{0} \cdot \nabla u_{0}+B \nabla u_{0} \cdot \nabla h_{\varepsilon}^{U}+\frac{1}{2} B \nabla h_{\varepsilon}^{U} \cdot \nabla h_{\varepsilon}^{U}\right)=\Phi^{\prime}(\xi) B \nabla u_{0} \cdot\left(\nabla \tilde{u}_{\varepsilon}-\nabla h_{\varepsilon}^{U}\right),
\end{aligned}
$$


and

$$
\left|\xi-\frac{1}{2} B \nabla u_{0} \cdot \nabla u_{0}-\frac{1}{2} B \nabla h_{\varepsilon}^{U} \cdot \nabla h_{\varepsilon}^{U}\right| \leq \max \left(\left|B \nabla u_{0} \cdot \nabla \tilde{u}_{\varepsilon}\right|,\left|B \nabla u_{0} \cdot \nabla h_{\varepsilon}^{U}\right|\right) .
$$

It comes

$$
\mathcal{E}_{4}^{21}(\varepsilon)=\int_{D_{1}} \beta_{\varepsilon}\left(\Phi^{\prime}(\xi)-\Phi^{\prime}\left(\frac{1}{2} B \nabla u_{0} \cdot \nabla u_{0}\right)\right) B \nabla u_{0} \cdot\left(\nabla \tilde{u}_{\varepsilon}-\nabla h_{\varepsilon}^{U}\right) \mathrm{d} x .
$$

The $W^{1, \infty}$ regularity of $u_{0}$ in $D_{1}$, the Cauchy-Schwarz inequality and the Lipschitz continuity of $\Phi^{\prime}$ yield:

$$
\left|\mathcal{E}_{4}^{21}(\varepsilon)\right| \leq c\left\|\xi-\frac{1}{2} B \nabla u_{0} \cdot \nabla u_{0}\right\|_{L^{2}\left(D_{1}\right)}\left\|\nabla \tilde{u}_{\varepsilon}-\nabla h_{\varepsilon}^{U}\right\|_{L^{2}\left(D_{1}\right)} .
$$

Using (5.4) we get:

$$
\left|\mathcal{E}_{4}^{21}(\varepsilon)\right| \leq c\left(\left\|B \nabla u_{0} \cdot \nabla \tilde{u}_{\varepsilon}\right\|_{L^{2}\left(D_{1}\right)}+\left\|B \nabla u_{0} \cdot \nabla h_{\varepsilon}^{U}\right\|_{L^{2}\left(D_{1}\right)}+\left\|\frac{1}{2} B \nabla h_{\varepsilon}^{U} \cdot \nabla h_{\varepsilon}^{U}\right\|_{L^{2}\left(D_{1}\right)}\right)\left\|\nabla \tilde{u}_{\varepsilon}-\nabla h_{\varepsilon}^{U}\right\|_{L^{2}\left(D_{1}\right)} .
$$

Using again the $W^{1, \infty}$ regularity of $u_{0}$ in $D_{1}$ together with Lemmas 5.1 and 5.2 we derive that $\mathcal{E}_{4}^{21}(\varepsilon)=$ $o\left(\varepsilon^{2}\right)$.

It stems from the Lipschitz continuity of $\Phi$ and Lemma 5.1 that

$$
\begin{aligned}
\mathcal{E}_{4}^{22}(\varepsilon)=\int_{D_{2}} \beta_{\varepsilon}\left[\Phi\left(\frac{1}{2} B \nabla u_{0} \cdot \nabla u_{0}+B \nabla u_{0} \cdot \nabla \tilde{u}_{\varepsilon}\right)-\right. & \Phi\left(\frac{1}{2} B \nabla u_{0} \cdot \nabla u_{0}+B \nabla u_{0} \cdot \nabla h_{\varepsilon}^{U}\right) \\
& \left.-\Phi^{\prime}\left(\frac{1}{2} B \nabla u_{0} \cdot \nabla u_{0}\right) B \nabla u_{0} \cdot\left(\nabla \tilde{u}_{\varepsilon}-\nabla h_{\varepsilon}^{U}\right)\right] \mathrm{d} x+O\left(\varepsilon^{4}\right) .
\end{aligned}
$$

This expression can be rewritten as:

$$
\begin{aligned}
& \mathcal{E}_{4}^{22}(\varepsilon)=\int_{D_{2}} \beta_{\varepsilon}\left[\Phi\left(\frac{1}{2} B \nabla u_{0} \cdot \nabla u_{0}+B \nabla u_{0} \cdot \nabla \tilde{u}_{\varepsilon}\right)-\Phi\left(\frac{1}{2} B \nabla u_{0} \cdot \nabla u_{0}\right)-\Phi^{\prime}\left(\frac{1}{2} B \nabla u_{0} \cdot \nabla u_{0}\right) B \nabla u_{0} \cdot \nabla \tilde{u}_{\varepsilon}\right] \mathrm{d} x \\
& -\int_{D_{2}} \beta_{\varepsilon}\left[\Phi\left(\frac{1}{2} B \nabla u_{0} \cdot \nabla u_{0}+B \nabla u_{0} \cdot \nabla h_{\varepsilon}^{U}\right)-\Phi\left(\frac{1}{2} B \nabla u_{0} \cdot \nabla u_{0}\right)-\Phi^{\prime}\left(\frac{1}{2} B \nabla u_{0} \cdot \nabla u_{0}\right) B \nabla u_{0} \cdot \nabla h_{\varepsilon}^{U}\right] \mathrm{d} x+O\left(\varepsilon^{4}\right) .
\end{aligned}
$$

Using the mean value theorem and the Lipschitz continuity of $\Phi^{\prime}$, it comes

$$
\left|\mathcal{E}_{4}^{22}(\varepsilon)\right| \leq c \int_{D_{2}}\left|B \nabla u_{0} \cdot \nabla \tilde{u}_{\varepsilon}\right|^{2} \mathrm{~d} x+c \int_{D_{2}}\left|B \nabla u_{0} \cdot \nabla h_{\varepsilon}^{V}\right|^{2} \mathrm{~d} x+c \varepsilon^{4} .
$$

The Cauchy-Schwarz inequality together with the assumption that $u_{0} \in W^{1,4}\left(D_{2}\right)$ yield

$$
\left|\mathcal{E}_{4}^{22}(\varepsilon)\right| \leq c\left\|\nabla u_{0}\right\|_{L^{4}\left(D_{2}\right)}^{2}\left\|\nabla \tilde{u}_{\varepsilon}\right\|_{L^{4}\left(D_{2}\right)}^{2}+c\left\|\nabla u_{0}\right\|_{L^{4}\left(D_{2}\right)}^{2}\left\|\nabla h_{\varepsilon}^{V}\right\|_{L^{4}\left(D_{2}\right)}^{2}+c \varepsilon^{4} .
$$

Using Lemmas 5.1 and 5.2, it comes $\mathcal{E}_{4}^{22}(\varepsilon)=o\left(\varepsilon^{2}\right)$. We conclude that $\mathcal{E}_{4}(\varepsilon)=o\left(\varepsilon^{2}\right)$.

(5) We use the notation

$$
V_{J 1}(\varepsilon)-\mathcal{E}_{4}(\varepsilon)=\int_{\tilde{D}} \beta_{\varepsilon}(y) F_{\varepsilon}(y, y) \mathrm{d} y
$$


with

$$
\begin{aligned}
F_{\varepsilon}(x, y)=\Phi\left(\frac{1}{2} B \nabla u_{0}(x) \cdot\right. & \left.\nabla u_{0}(x)+B \nabla u_{0}(x) \cdot \nabla h_{\varepsilon}^{U}(y)+\frac{1}{2} B \nabla h_{\varepsilon}^{U}(y) \cdot \nabla h_{\varepsilon}^{U}(y)\right) \\
& -\Phi\left(\frac{1}{2} B \nabla u_{0}(x) \cdot \nabla u_{0}(x)\right)-\Phi^{\prime}\left(\frac{1}{2} B \nabla u_{0}(x) \cdot \nabla u_{0}(x)\right) B \nabla u_{0}(x) \cdot \nabla h_{\varepsilon}^{U}(y) .
\end{aligned}
$$

We have in this way

$$
\mathcal{E}_{5}(\varepsilon)=\int_{\tilde{D}} \beta_{\varepsilon} F_{\varepsilon}(y, y) \mathrm{d} y-\int_{\mathbb{R}^{2}} \beta_{\varepsilon}^{*} F_{\varepsilon}\left(x_{0}, y\right) \mathrm{d} y .
$$

Next, we choose $R \in(0, \eta]$ such that $\beta_{0}$ and $\beta_{1}$ are constant in $B\left(x_{0}, R\right)$. We split (5.6) as

$$
\mathcal{E}_{5}(\varepsilon)=\int_{B\left(x_{0}, R\right)} \beta_{\varepsilon}^{*} F_{\varepsilon}(y, y) \mathrm{d} y+\int_{\tilde{D} \backslash \overline{B\left(x_{0}, R\right)}} \beta_{\varepsilon} F_{\varepsilon}(y, y) \mathrm{d} y-\int_{B\left(x_{0}, R\right)} \beta_{\varepsilon}^{*} F_{\varepsilon}\left(x_{0}, y\right) \mathrm{d} y-\int_{\mathbb{R}^{2} \backslash \overline{B\left(x_{0}, R\right)}} \beta_{\varepsilon}^{*} F_{\varepsilon}\left(x_{0}, y\right) \mathrm{d} y .
$$

We get $\mathcal{E}_{5}(\varepsilon)=\mathcal{E}_{5}^{1}(\varepsilon)+\mathcal{E}_{5}^{2}(\varepsilon)+\mathcal{E}_{5}^{3}(\varepsilon)$ with

$$
\begin{gathered}
\mathcal{E}_{5}^{1}(\varepsilon)=\int_{B\left(x_{0}, R\right)} \beta_{\varepsilon}^{*}\left[F_{\varepsilon}(y, y)-F_{\varepsilon}\left(x_{0}, y\right)\right] \mathrm{d} y \\
\mathcal{E}_{5}^{2}(\varepsilon)=\int_{\tilde{D} \backslash \frac{B\left(x_{0}, R\right)}{}} \beta_{\varepsilon} F_{\varepsilon}(y, y) \mathrm{d} y, \quad \mathcal{E}_{5}^{3}(\varepsilon)=-\int_{\mathbb{R}^{2} \backslash \frac{}{B\left(x_{0}, R\right)}} \beta_{\varepsilon}^{*} F_{\varepsilon}\left(x_{0}, y\right) \mathrm{d} y .
\end{gathered}
$$

A change of variable yields (we recall that $x_{0}$ is at the origin):

$$
\mathcal{E}_{5}^{1}(\varepsilon)=\varepsilon^{2} \int_{\mathbb{R}^{2}} \chi_{B(0, R)}(\varepsilon x) \beta_{\varepsilon}^{*}(\varepsilon x)\left[F_{\varepsilon}(\varepsilon x, \varepsilon x)-F_{\varepsilon}\left(x_{0}, \varepsilon x\right)\right] \mathrm{d} x .
$$

From (5.5) and the regularity assumptions, we obtain that

$$
\lim _{\varepsilon \rightarrow 0} \chi_{B(0, R)}(\varepsilon x) \beta_{\varepsilon}^{*}(\varepsilon x)\left[F_{\varepsilon}(\varepsilon x, \varepsilon x)-F_{\varepsilon}\left(x_{0}, \varepsilon x\right)\right]=0 \quad \forall x \in \mathbb{R}^{2} .
$$

Now we write the decomposition

$$
\begin{aligned}
F_{\varepsilon}(x, y)= & {\left[\Phi\left(\frac{1}{2} B \nabla u_{0}(x) \cdot \nabla u_{0}(x)+B \nabla u_{0}(x) \cdot \nabla h_{\varepsilon}^{U}(y)+\frac{1}{2} B \nabla h_{\varepsilon}^{U}(y) \cdot \nabla h_{\varepsilon}^{U}(y)\right)\right.} \\
& \left.-\Phi\left(\frac{1}{2} B \nabla u_{0}(x) \cdot \nabla u_{0}(x)+B \nabla u_{0}(x) \cdot \nabla h_{\varepsilon}^{U}(y)\right)\right] \\
& +\left[\Phi\left(\frac{1}{2} B \nabla u_{0}(x) \cdot \nabla u_{0}(x)+B \nabla u_{0}(x) \cdot \nabla h_{\varepsilon}^{U}(y)\right)\right. \\
& \left.-\Phi\left(\frac{1}{2} B \nabla u_{0}(x) \cdot \nabla u_{0}(x)\right)-\Phi^{\prime}\left(\frac{1}{2} B \nabla u_{0}(x) \cdot \nabla u_{0}(x)\right) B \nabla u_{0}(x) \cdot \nabla h_{\varepsilon}^{U}(y)\right] .
\end{aligned}
$$

The Lipschitz continuity of $\Phi$ and $\Phi^{\prime}$ yields

$$
\left|F_{\varepsilon}(x, y)\right| \leq c\left|\nabla h_{\varepsilon}^{U}(y)\right|^{2}+c\left|\nabla u_{0}(x)\right|^{2}\left|\nabla h_{\varepsilon}^{U}(y)\right|^{2} \quad \forall x, y \in \tilde{D} .
$$

Using the regularity of $u_{0}$ in $B\left(x_{0}, R\right)$, it follows that

$$
\left|F_{\varepsilon}(\varepsilon x, \varepsilon x)\right|+\left|F_{\varepsilon}\left(x_{0}, \varepsilon x\right)\right| \leq c\left|\nabla h_{\varepsilon}^{U}(\varepsilon x)\right|^{2}=c\left|H_{\rho}^{U}(x)\right|^{2} \quad \forall x \in B(0, R / \varepsilon) .
$$



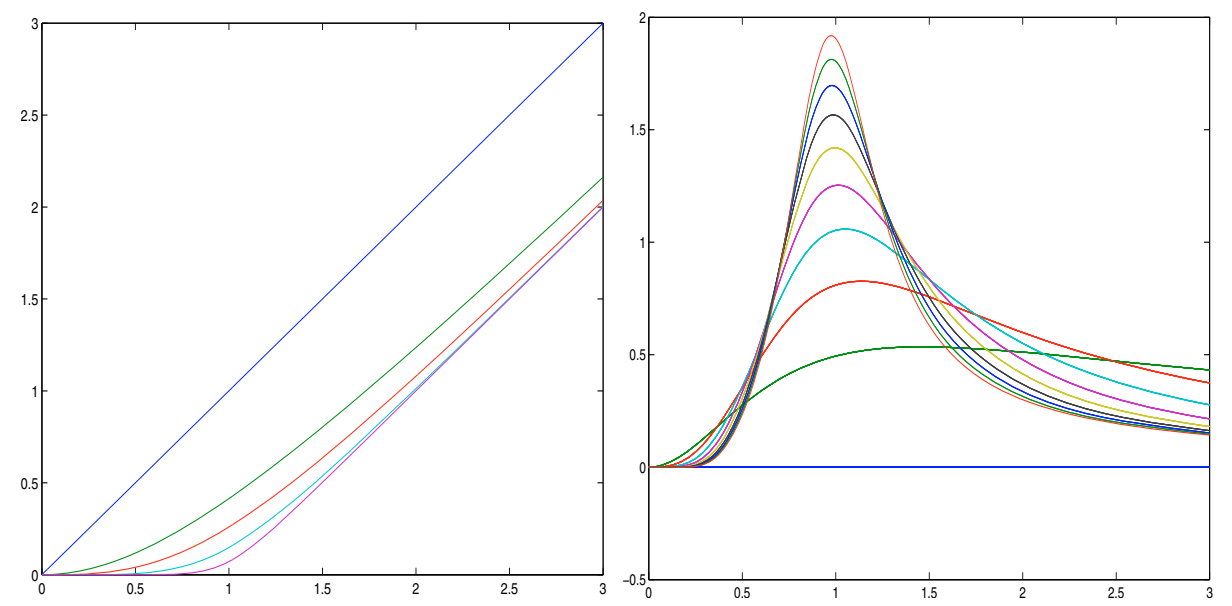

Figure 1. Left: graph of the function $\Theta_{p}$ for $p=1,2,3,5,10$, respectively (from top to bottom); right: graph of the function $\hat{\Psi}_{ \pm 1}$ for $p=1, \ldots, 10$, respectively (from bottom to top at $t=1)$.

The function $H_{\rho}^{U}$ is defined by (4.17) in $\mathbb{R}^{2} \backslash \bar{\omega}$ and constant in $\omega$. Therefore $\left|H_{\rho}^{U}\right|^{2}$ is integrable over $\mathbb{R}^{2}$. In consequence of this and (5.7), the Lebesgue dominated convergence theorem yields $\mathcal{E}_{5}^{1}(\varepsilon)=o\left(\varepsilon^{2}\right)$. We bound $\mathcal{E}_{5}^{2}(\varepsilon)$ with the help of (5.8) and the Cauchy-Schwarz inequality. We obtain

$$
\left|\mathcal{E}_{5}^{2}(\varepsilon)\right| \leq c\left\|\nabla h_{\varepsilon}^{U}\right\|_{L^{2}\left(\tilde{D} \backslash \overline{B\left(x_{0}, R\right)}\right)}^{2}+c\left\|\nabla u_{0}\right\|_{L^{4}\left(\tilde{D} \backslash \overline{B\left(x_{0}, R\right)}\right)}^{2}\left\|\nabla h_{\varepsilon}^{U}\right\|_{L^{4}\left(\tilde{D} \backslash \overline{B\left(x_{0}, R\right)}\right)}^{2} .
$$

Then Lemma 5.1 provides $\mathcal{E}_{5}^{2}(\varepsilon)=O\left(\varepsilon^{4}\right)$. In a similar way we obtain that $\mathcal{E}_{5}^{3}(\varepsilon)=O\left(\varepsilon^{4}\right)$.

(6) When $\varepsilon$ is sufficiently small, we have

$$
\mathcal{E}_{6}(\varepsilon)=\left(\beta_{1}^{*}-\beta_{0}^{*}\right) \int_{\omega_{\varepsilon}}\left[\Phi\left(\frac{1}{2} B \nabla u_{0}(x) \cdot \nabla u_{0}(x)\right)-\Phi\left(\frac{1}{2} B \nabla u_{0}\left(x_{0}\right) \cdot \nabla u_{0}\left(x_{0}\right)\right)\right] \mathrm{d} x .
$$

Using the Lipschitz continuity of $\Phi$ and the $\alpha$-Hölder continuity of $\nabla u_{0}$ in the vicinity of $x_{0}$, we derive that $\mathcal{E}_{6}(\varepsilon)=O\left(\varepsilon^{2+\alpha}\right)$. This completes the proof of Theorem 4.3.

\section{EXAmple Of PENALTy FUnCTiON}

Given a real parameter $p \geq 1$, we consider the penalty function:

$$
\Phi_{p}(t)=\Theta_{p}\left(\frac{t}{M}\right)
$$

with

$$
\begin{aligned}
\Theta_{p}: \mathbb{R}_{+} & \rightarrow \mathbb{R}_{+}, \\
t & \mapsto\left(1+t^{p}\right)^{1 / p}-1 .
\end{aligned}
$$

The function $\Theta_{p}$ is plotted in Figure 1. It is straightforward to check that $\Theta_{p}$ satisfies the assumptions of Theorem 4.3 for every $p \geq 1$. Furthermore, when $p$ goes to infinity, $\Theta_{p}$ converges uniformly on $\mathbb{R}_{+}$to the non-differentiable function:

$$
\Theta_{\infty}(t)= \begin{cases}0 & \text { if } t \leq 1 \\ t-1 & \text { if } t \geq 1\end{cases}
$$


The function $\hat{\Psi}_{\rho}$ associated with $\Theta_{p}$ is also represented in Figure 1 for $\rho= \pm 1$ and $p$ varying from 1 to 10 . Actually, when condition (2.1) is fulfilled, only the values of $\hat{\Psi}_{\rho}$ for $\rho=\frac{\alpha_{\text {in }}-\alpha_{\text {out }}}{\alpha_{\text {in }}+\alpha_{\text {out }}} \approx 1$ and $\rho=\frac{\alpha_{\text {out }}-\alpha_{\text {in }}}{\alpha_{\text {out }}+\alpha_{\text {in }}} \approx-1$ are of interest. The double integral (4.24) has been computed by a quadrature method.

\section{Algorithm}

We come back to the problem described in Section 2. As said in Section 2.2, the proposed algorithm is based on the conjecture that local minimizers of $(2.4)$ are close (because $\beta_{\text {out }} \neq 0$ ) to local minimizers of the problem

$$
\min _{\Omega \in \mathcal{E}} I_{\Omega}\left(u_{\Omega}\right)+\gamma \int_{\tilde{D}} \beta_{\Omega} \Phi_{\infty}\left(\frac{1}{2} B \nabla u_{\Omega} \cdot \nabla u_{\Omega}\right) \mathrm{d} x \quad \text { subject to }(2.2)
$$

provided that $\gamma$ is large enough. Because $\Phi_{\infty}$ does not fulfill the assumptions of Theorem 4.3, we replace $\Phi_{\infty}$ by $\Phi_{p}$ and let $p$ go to infinity. The algorithm reads as follows.

(1) Choose $\gamma$ large enough, an increasing sequence $\left(p_{n}\right)_{n \in \mathbb{N}}$ of positive numbers tending to infinity and an initial domain $\Omega_{0} \in \mathcal{E}$. Set $n=0$.

(2) Iterate:

- Starting from the domain $\Omega_{n}$, find $\Omega_{n+1}$ (local) solution of the problem

$$
\min _{\Omega \in \mathcal{E}} I_{\Omega}\left(u_{\Omega}\right)+\gamma \int_{\tilde{D}} \beta_{\Omega} \Phi_{p_{n}}\left(\frac{1}{2} B \nabla u_{\Omega} \cdot \nabla u_{\Omega}\right) \mathrm{d} x \quad \text { subject to }(2.2) ;
$$

- Increment $n \leftarrow n+1$.

\section{NUMERICAL RESULTS}

In all the computations we take the conductivity coefficients $\alpha_{\text {in }}=\beta_{\text {in }}=1$ and $\alpha_{\text {out }}=\beta_{\text {out }}=10^{-5}$. The objective functional to be minimized is

$$
I_{\Omega}\left(u_{\Omega}\right)=|\Omega|+\lambda K_{\Omega}\left(u_{\Omega}\right)
$$

where $|\Omega|$ is the Lebesgue measure of $\Omega$ and $K_{\Omega}\left(u_{\Omega}\right)$ is the energy

$$
K_{\Omega}\left(u_{\Omega}\right)=\int_{D} \alpha_{\Omega}\left|\nabla u_{\Omega}\right|^{2} \mathrm{~d} x=\int_{\Gamma_{N}} g u_{\Omega} \mathrm{d} s
$$

The topological sensitivity of $|\Omega|$ is obvious, and, using the notations of Theorem 4.3, that of the energy functional is (see [6] or apply Thm. 4.3 with $\Phi=2 \mathrm{Id}, B=I, \tilde{D}=D$ and $\beta_{\Omega}=\alpha_{\Omega}$ ):

$$
K_{\Omega_{\varepsilon}}\left(u_{\Omega_{\varepsilon}}\right)-K_{\Omega}\left(u_{\Omega}\right)=-2 \pi \varepsilon^{2} \alpha_{\Omega}\left(x_{0}\right) \rho\left|\nabla u_{\Omega}\left(x_{0}\right)\right|^{2}+o\left(\varepsilon^{2}\right) .
$$

We consider an isotropic constraint $(B=I)$ over the whole domain $(\tilde{D}=D)$. We apply the algorithm described in Section 7 with $p_{n}=8 \times 2^{n}$, as it turns out in the proposed examples that starting with $p_{0}=8$ does not raise any numerical difficulty and allows a gain of time. The internal minimization problem (7.1) is solved using the algorithm described in [7], which is based on a level-set domain representation and successive approximations of topological optimality conditions. We assume that $\mathcal{E}$ contains all the domains which can be obtained in this way. Since this procedure requires many evaluations of $\hat{\Psi}_{ \pm 1}$, this function is tabulated for each value of $p$, with the sampling points concentrated at the locations of sharp variations. In every example, we take $M=2$ 

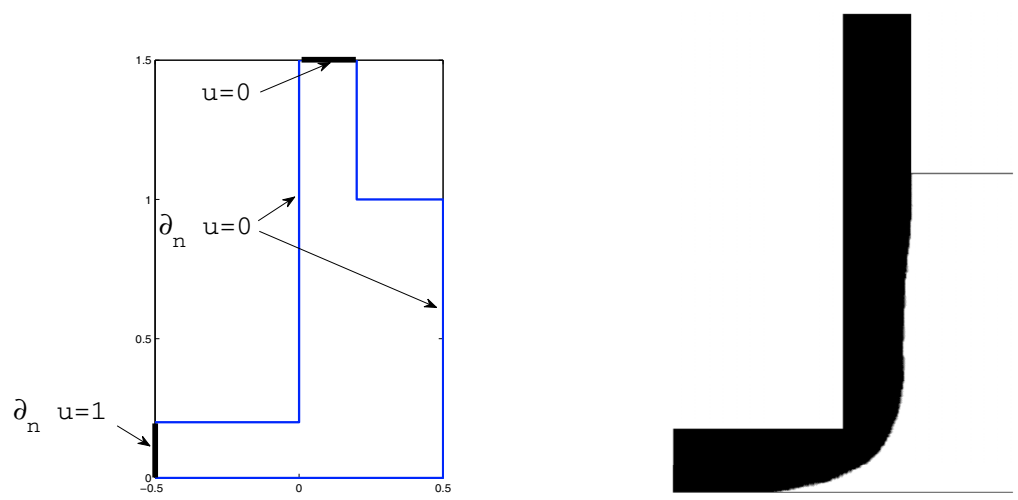

Figure 2. Computational domain and boundary conditions for the L-shaped conductor (left), unconstrained solution (right).

TABLE 1. L-shaped conductor: data obtained after 3 iterations.

\begin{tabular}{|c|c|c|c|}
\hline$\gamma$ & $Q\left(\Omega^{\sharp}\right)$ & $I\left(\Omega^{\sharp}\right)$ & CPU time $(\mathrm{s})$ \\
\hline \hline $10^{2}$ & 1.5225 & 0.7777 & 193 \\
\hline $10^{3}$ & 1.0807 & 0.7899 & 249 \\
\hline $10^{4}$ & 1.0177 & 0.7922 & 387 \\
\hline $10^{5}$ & 0.9615 & 0.8002 & 468 \\
\hline
\end{tabular}

and a full domain initialization, i.e. $\Omega_{0}=D$. In order to measure a possible constraint violation, we will compute the number

$$
Q(\Omega)=\max _{x \in \Omega} \sqrt{\frac{\frac{1}{2}\left|\nabla u_{\Omega}(x)\right|^{2}}{M}} .
$$

The computations are performed with the help of the PDE Toolbox of Matlab which generates $P 1$ finite elements.

\subsection{L-shaped conductor}

We consider as first example the domain depicted in Figure 2 (left) and the objective functional (8.1). We use a regular mesh containing 36241 nodes. For comparison, we first solve the unconstrained problem $(\gamma=0)$ with $\lambda=1$ (see Fig. 2, right). As expected, the singularity of the solution at the re-entrant corner is preserved.

\subsubsection{Influence of the penalty coefficient $\gamma$}

We now run the algorithm with the same multiplier $\lambda=1$ and different values of $\gamma$. Each time, we perform 3 iterations, corresponding to the parameters $p_{0}=8, p_{1}=16$ and $p_{2}=32$. Some numerical data related to the final domain $\Omega^{\sharp}$ are reported in Table 1. Unsurprisingly, $Q\left(\Omega^{\sharp}\right)$ decreases as $\gamma$ increases, whereas the computer time increases. This computer time is measured on a standard PC equipped with a $2.4 \mathrm{GHz}$ processor. We notice that the constraint is almost fulfilled for $\gamma=10^{4}$. When $\gamma=10^{5}$, the bound $M$ is not attained at iteration 3, but we can get $Q\left(\Omega^{\sharp}\right)$ closer to 1 by continuing the iterations. For instance, we obtain $Q\left(\Omega^{\sharp}\right)=0.9945$ by iterating up to $p=256$.

In view of these results, we now fix $\gamma=10^{4}$. This value will be used in all the computations presented in the sequel.

\subsubsection{Influence of the number of iterations}

The domains obtained at the end of some selected iterations are shown in Figure 3. We observe that there is nearly no more evolution after iteration $3(p=32)$. 

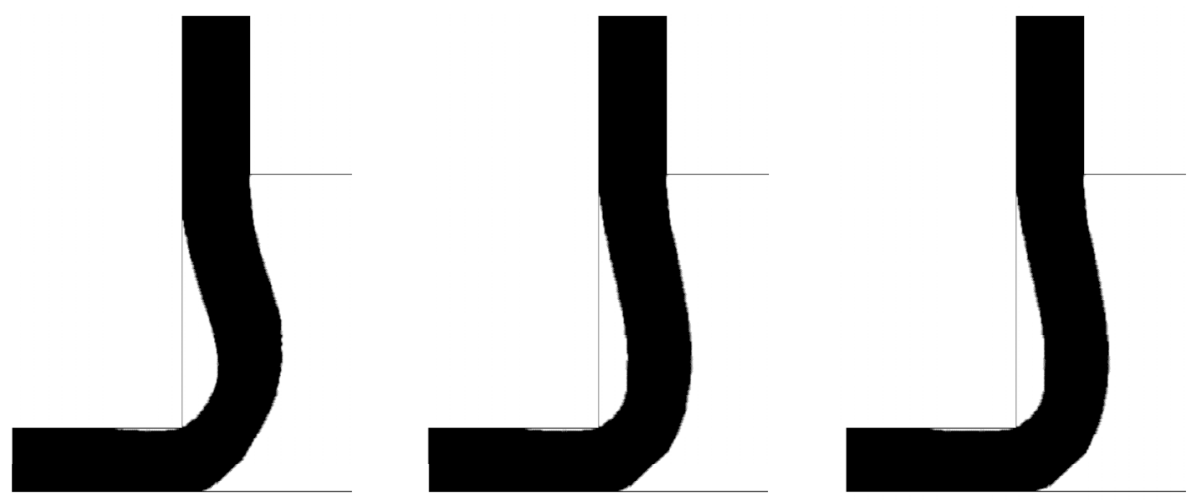

FIgURE 3. L-shaped conductor with $\lambda=1$ and $\gamma=10^{4}$ : snapshots for $p=8, p=32$ and $p=128$ (from left to right).

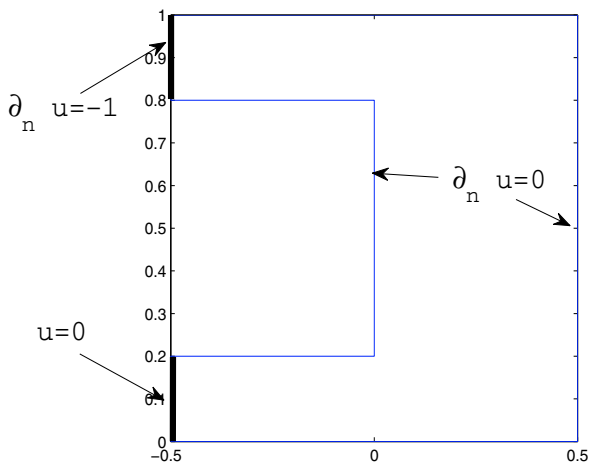

Figure 4. Computational domain for the U-shaped conductor.

\subsection{U-shaped conductor}

We consider now an U-shaped conductor (see Fig. 4), discretized with the help of 36241 nodes. The domain obtained when solving the unconstrained problem with $\lambda=1$ is depicted in Figure 5 , left. Then we run two computations with the constraint (see Fig. 5, middle and right). Each time we perform 3 iterations (up to $p=32)$.

(1) Case 1: $\lambda=1$. The obtained domain $\Omega^{\sharp}$ satisfies $Q\left(\Omega^{\sharp}\right)=1.0028$.

(2) Case 2: $\lambda=0.5$. We arrive at $Q\left(\Omega^{\sharp}\right)=0.9884$.

\subsection{S-shaped conductor}

The description and the obtained domain $\Omega^{\sharp}$ for this problem are given in Figure 6 . We have taken $\lambda=0.5$, a mesh with 15641 nodes, and performed 5 iterations (up to $p=128$ ), which used 204 s of CPU time. We have got $Q\left(\Omega^{\sharp}\right)=0.9905$.

\subsection{X-shaped conductor}

We consider a last example (see Fig. 7) for which topology changes actually occur. The results obtained with $\lambda=1$ and a mesh of 20769 nodes in the unconstrained and constrained cases are shown in Figure 8, left and middle. For this latter case we have performed 5 iterations and we have got $Q\left(\Omega^{\sharp}\right)=0.991$. We notice 

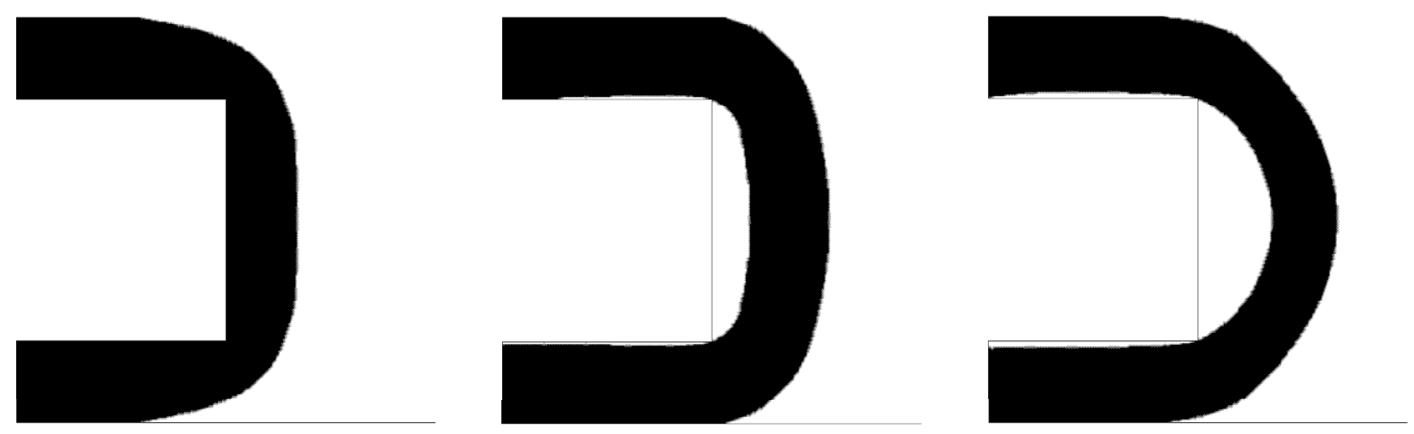

FigurE 5. Final design for the U-shaped conductor: unconstrained case $(\lambda=1$, left), case 1 $(\lambda=1$, middle), case $2(\lambda=0.5$, right $)$.
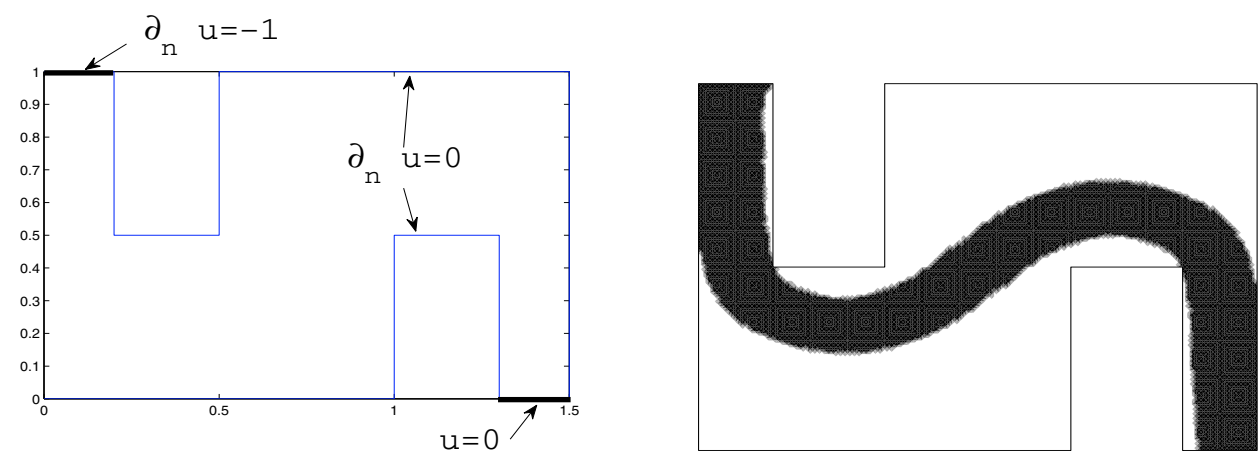

Figure 6. S-shaped conductor.

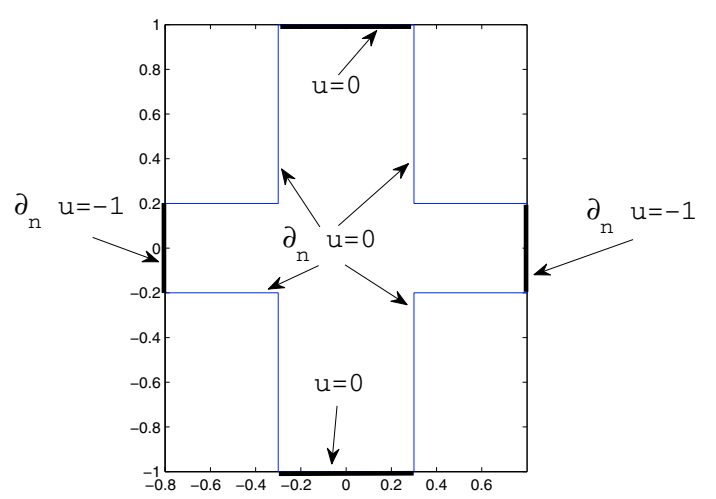

Figure 7. Computational domain for the X-shaped conductor.

that the two solutions do not have the same topology. Then we have performed the same computation on a finer mesh (82 497 nodes, see Fig. 8, right, $\left.Q\left(\Omega^{\sharp}\right)=1.0014\right)$.

\subsection{Conclusion}

In contrast to standard algorithms based only on energy minimization, the proposed algorithm performs a pointwise control of the state, and consequently avoids undesirable geometric singularities. Such singularities 

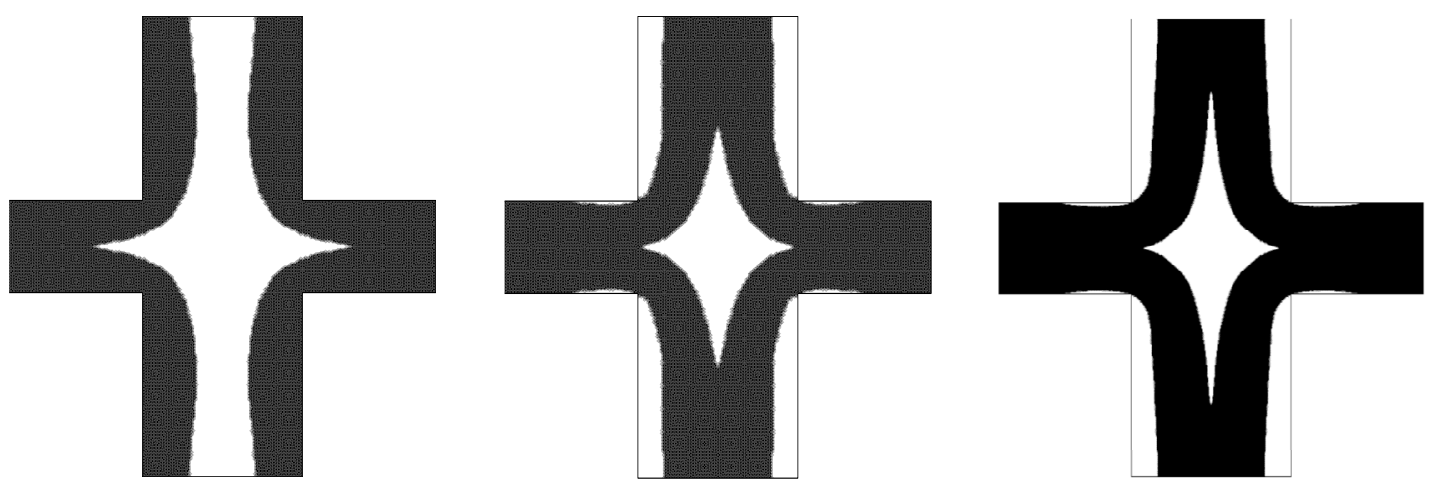

FiguRE 8. Unconstrained (left) and constrained (middle for the first mesh, right for the second mesh) solutions for the X-shaped conductor.

are allowed to be present at the initialization since the domain is not bound to stay in the feasible set during the iterations. In return, the constraint can be slightly violated when the algorithm is stopped. This shortcoming can be easily bypassed by choosing $M$ slightly lower than the targeted value. As already observed in classical optimization, the choice of the penalization coefficient $\gamma$ is a matter of compromise between the risk of significant violation of the constraint if $\gamma$ is too small and the ill-conditioning of the internal minimization problem (7.1) if $\gamma$ is too large, resulting in an increase of the computer cost. In practice, a few tries lead to a suitable value. Finally, we point out that we have always used a regular and relatively fine mesh in order to capture the singularities without influencing the optimization, but the use of a locally refined mesh, constructed in an appropriate way at the beginning or adaptive, could significantly reduce the computational effort.

\section{REFERENCES}

[1] R.A. Adams, Sobolev spaces, Pure and Applied Mathematics 65. Academic Press [A subsidiary of Harcourt Brace Jovanovich, Publishers], New York-London (1975).

[2] G. Allaire, Shape optimization by the homogenization method, Applied Mathematical Sciences 146. Springer-Verlag, New York (2002).

[3] G. Allaire, F. Jouve and H. Maillot, Topology optimization for minimum stress design with the homogenization method. Struct. Multidiscip. Optim. 28 (2004) 87-98.

[4] G. Allaire, F. Jouve and A.-M. Toader, Structural optimization using sensitivity analysis and a level-set method. J. Comput. Phys. 194 (2004) 363-393.

[5] G. Allaire, F. de Gournay, F. Jouve and A.-M. Toader, Structural optimization using topological and shape sensitivity via a level set method. Control Cybern. 34 (2005) 59-80.

[6] S. Amstutz, Sensitivity analysis with respect to a local perturbation of the material property. Asymptot. Anal. 49 (2006) 87-108.

[7] S. Amstutz and H. Andrä, A new algorithm for topology optimization using a level-set method. J. Comput. Phys. 216 (2006) 573-588.

[8] J. Appell and P.P. Zabrejko, Nonlinear superposition operators, Cambridge Tracts in Mathematics 95. Cambridge University Press, Cambridge (1990).

[9] M.P. Bendsøe and N. Kikuchi, Generating optimal topologies in structural design using a homogenization method. Comput. Methods Appl. Mech. Engrg. 71 (1988) 197-224.

[10] M.P. Bendsøe and O. Sigmund, Topology optimization, Theory, methods and applications. Springer-Verlag, Berlin (2003).

[11] J.F. Bonnans, J.C. Gilbert, C. Lemaréchal and C.A. Sagastizábal, Numerical optimization, Theoretical and practical aspects. Universitext, Springer-Verlag, Berlin, Second Edition (2006).

[12] M. Burger and R. Stainko, Phase-field relaxation of topology optimization with local stress constraints. SIAM J. Control Optim. 45 (2006) 1447-1466 (electronic).

[13] M. Burger, B. Hackl and W. Ring, Incorporating topological derivatives into level set methods. J. Comput. Phys. 194 (2004) 344-362.

[14] P. Duysinx and M.P. Bendsøe, Topology optimization of continuum structures with local stress constraints. Internat. J. Numer. Methods Engrg. 43 (1998) 1453-1478. 
[15] H. Eschenauer, V.V. Kobolev and A. Schumacher, Bubble method for topology and shape optimization of structures. Struct. Optimization 8 (1994) $42-51$.

[16] S. Garreau, P. Guillaume and M. Masmoudi, The topological asymptotic for PDE systems: the elasticity case. SIAM J. Control Optim. 39 (2001) 1756-1778 (electronic).

[17] D. Gilbarg and N.S. Trudinger, Elliptic partial differential equations of second order, Classics in Mathematics. Springer-Verlag, Berlin (2001). Reprint of the 1998 edition.

[18] P. Grisvard, Elliptic problems in nonsmooth domains, Monographs and Studies in Mathematics 24. Pitman (Advanced Publishing Program), Boston, USA (1985).

[19] A. Henrot and M. Pierre, Variation et optimisation de formes, Mathématiques et applications 48. Springer-Verlag, Heidelberg (2005).

[20] M. Hintermüller and K. Kunisch, Stationary optimal control problems with pointwise state constraints (to appear).

[21] M. Hintermüller and W. Ring, A level set approach for the solution of a state-constrained optimal control problem. Numer. Math. 98 (2004) 135-166.

[22] K. Ito and K. Kunisch, Semi-smooth Newton methods for state-constrained optimal control problems. Systems Control Lett. 50 (2003) 221-228.

[23] C. Meyer, A. Rösch and F. Tröltzsch, Optimal control of PDEs with regularized pointwise state constraints. Comput. Optim. Appl. 33 (2006) 209-228.

[24] F. Murat and J. Simon, Étude de problèmes d'optimal design, in Lecture Notes in Computer Sciences 41, Springer-Verlag, Berlin (1976) 54-62.

[25] S.A. Nazarov and J. Sokołowski, Asymptotic analysis of shape functionals. J. Math. Pures Appl. 82 (2003) 125-196.

[26] J.A. Norato, M.P. Bendsøe, R.B. Haber and D.A. Tortorelli, A topological derivative method for topology optimization. Struct. Multidiscip. Optim. 33 (2007) 375-386.

[27] M. Petzoldt, Regularity results for Laplace interface problems in two dimensions. Z. Anal. Anwendungen 20 (2001) $431-455$.

[28] J.-J. Rückmann and J.A. Gómez, On generalized semi-infinite programming. Top 14 (2006) 1-59.

[29] J.J. Rückmann and A. Shapiro, First-order optimality conditions in generalized semi-infinite programming. J. Optim. Theory Appl. 101 (1999) 677-691.

[30] G. Savaré, Regularity results for elliptic equations in Lipschitz domains. J. Funct. Anal. 152 (1998) 176-201.

[31] J. Simon, Differentiation with respect to the domain in boundary value problems. Numer. Funct. Anal. Optim. 2 (1980) 649-687.

[32] J. Sokołowski and A. Żochowski, On the topological derivative in shape optimization. SIAM J. Control Optim. 37 (1999) 1251-1272 (electronic).

[33] J. Sokołowski and J.-P. Zolésio, Introduction to shape optimization - Shape sensitivity analysis, Springer Series in Computational Mathematics 16. Springer-Verlag, Berlin (1992).

[34] G. Still, Generalized semi-infinite programming: numerical aspects. Optimization 49 (2001) 223-242.

[35] M.Y. Wang, X. Wang and D. Guo, A level set method for structural topology optimization. Comput. Methods Appl. Mech. Engrg. 192 (2003) 227-246. 